\title{
ANÁLISE DE VARIÂNCIA PARA DADOS DE TONALIDADE DE COR: UM CASO DE DADOS CIRCULARES
}

\section{ANTONIO WILLIAMS MOITA}

Bacharel em Matemática

Orientador: Prof. Dr. SILVIO SANDOVAL ZOCCHI

Dissertação apresentada à Escola Superior de Agricultura "Luiz de Queiroz", Universidade de São Paulo, para obtenção do título de Mestre em Agronomia, Área de Concentração: Estatística e Experimentação Agronômica.

P I R A C I C A B A

Estado de São Paulo - Brasil

Outubro - 2004 
Dados Internacionais de Catalogaçāo na Publicaçāo (CIP) DIVISÃO DE BIBLIOTECA E DOCUMENTAÇÃO - ESALQIUSP

Moita, Antonio Williams

Análise de variância para dados de tonalidade de cor : um caso de dados circulares I Antonio Williams Moita. - Piracicaba, 2004.

$50 \mathrm{p}$.

Dissertação (Mestrado) - - Escola Superior de Agricultura Luiz de Queiroz, 2004.

Bibliografia.

1. Alimentos:2. Análise de variância 3. Corl. Titulo :

CDD 519.535

"Permitida a cópia total ou parcial deste documento, desde que citada a fonte - 0 autor" 


\section{DEDICATÓRIA}

A

\section{DEUS}

pela oportunidade da vida.

Aos meus pais,

José Euclides Moita e

Francisca do Carmo Lima,

(in memoriam), por embalarem meus anseios e inspirarem minhas melhores realizações.

À minha amada esposa Cris, pelo seu amor, seu carinho, sua dedicação e sua compreeñão.

Aos filhos, Thiago \& Tatiana e netos :Raphael \& Yago minha fonte de inspiração e de alegria.

Aos irmãos Vilany, Ydália, Sebastião, Francisco, Manoel e Salette, meus primeiros mestres.

dedico. 


\section{AGRADECIMENTOS}

À Empresa Brasileira de Pesquisa Agropecuária (EMBRAPA), o suporte e o auxílio financeiro durante a realização do curso, nas pessoas de meus superiores José Amauri Buso, Waldir Aparecido Marouelli, Osmar Alves Carrijo, Ruy Rezende Fontes e do orientador acadêmico Welington Pereira.

À ESALQ/USP a oportunidade de realização do curso.

Ao professor Doutor Silvio Sandoval Zocchi, a paciência, a amizade e a orientação segura durante a realização de todo este trabalho.

À professora Doutora Clarice Garcia Borges Demétrio, coordenadora do curso, sua dedicação e seus valiosos conselhos.

Aos professores Décio Barbin, Roseli Aparecida Leandro, Sônia Maria de Stefano Piedade e Antonio Augusto Franco Garcia os valiosos ensinamentos ministrados.

Aos professores Carlos Tadeu dos Santos Dias, Edwin Moisés Marcos Ortega e Gabriel Adrián Sarriés, o convívio e a amizade.

Aos funcionários Solange Sabadin, Luciane Brajão, Jorge Wiendel e Rosa Maria Alves, a solicitude demonstrada e os auxílios prestados.

À Evelise M. Moda, a permissão para o uso dos dados de sua dissertação de mestrado.

Ao Dr. Albano S. Pereira, a sua cooperação na revisão de colorimetria.

Ao professor da UFC, João Maurício Araújo Mota, sua amizade e seus ensinamentos.

Ao professor doutor Bryan Manly, por sua revisão do resumo em inglês desta dissertação. 
Aos colegas de curso, Juliana Garcia Cespedes, Ana Alice Pillon, Carlos Henrique Garcia, Alda Zanin, Adriano Borgatto, Ana Maria Souza de Araújo, Silvano Cesar da Costa, Osmir Lavoranti, Suely Giolo, Maria Cristina Neves, Antonio Carlos Pontes, Melissa Oda, Helio Rubens, Júlio Pereira, Francine Ragonha, Alfredo Wenzel, William Saconato, Elisabeth Strapasson, Geneville Bergamo, David Miquelluti, Denise Viola, Renato R. Lima, Édila Cristina Souza, Angela Coelho, Lúcio Borges de Araújo, Osmar Jesus Macedo, Telde Natel Custódio, Elizabeth Regina, Luciana Pagliosa Carvalho, Ezequiel e Ramiro, a amizade e o companheirismo.

A todos os colegas da EMBRAPA-Hortaliças, em especial: Ossami Furomoto, Leonardo de Brito Giordano, Jairo Vidal Vieira, Manoel V. Mesquita Filho, Geni Litvin Villas Boas, Milza Moreira Lana, João Maria Charchar, Carlos Alberto Lopes, Gilmar Paulo Henz, João Bosco C. da Silva, Cláudia Silva da Costa Ribeiro, Sieglinde Brune, Alice Maria Quezado Duval, Antonio Francisco Souza, Cristina Maria M. Machado, Patrícia Silva Ritschel, Fausto Francisco dos Santos, Rita de Fátima A. Luengo, Henoque Ribeiro da Silva, Tereza Cristina de O. Saminez, Nirlene Junqueira Vilela, José Flávio Lopes, Paulo Mello, Leonardo Silva Boiteux, Warley Nascimento, Antonio Carlos Torres, Adonai Gimenez Calbo, Dione Melo da Silva, Neville V. Barbosa dos Reis, André Nepomuceno Dusi, Antônio Carlos de Ávila, Assis Marinho, Dejoel B. Lima, José L. Mendonça, Nozomu Makishima, Sabrina Isabel C. Carvalho e Rosane Parmagnani com os quais desenvolvi trabalhos de pesquisa na Embrapa Hortaliças.

Aos colegas da Área de Métodos Quantitativos da EMBRAPA, Geraldo da Silva e Souza, Lúcio José Vivaldi, Célia Maria Torres Cordeiro, Antonio Carlos Gomes e Fernando Luís Garagorry Cassales, a amizade e o aconselhamento técnico.

Aos colegas da área administrativa da EMBRAPA Hortaliças Sulamita, Paulo Fraga, Alfredo, Graça, Orébio, Regina e Fátima.

A todos aqueles que de alguma maneira, contribuíram para a realização deste trabalho. 


\section{SUMÁRIO}

\section{Página}

LISTA DE FIGURAS . . . . . . . . . . . . . . . . vii LISTA DE TABELAS .......................... ix

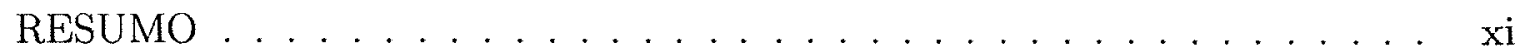

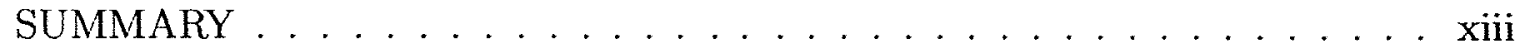

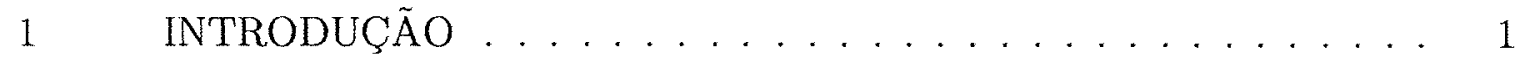

2 REVISÃO DE LITERATURA . . . . . . . . . . . . 3

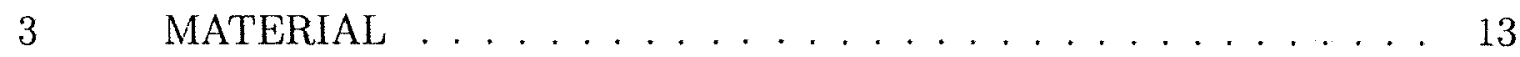

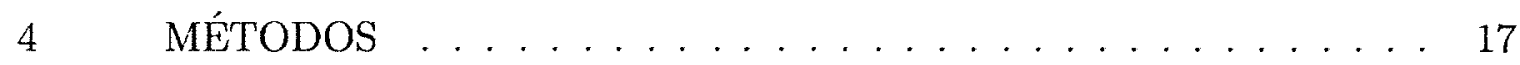

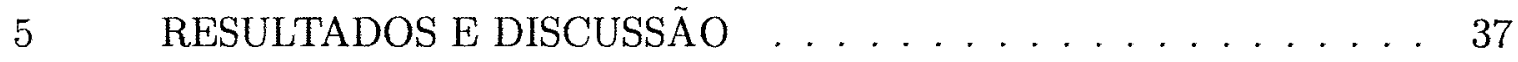

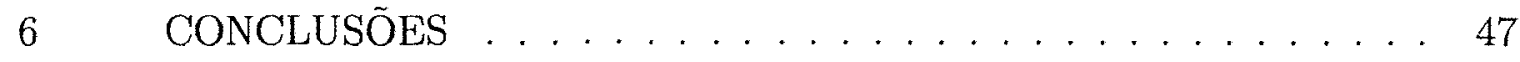

REFERÊNCIAS BIBLIOGRÁFICAS . . . . . . . . . . 48 


\section{LISTA DE FIGURAS}

Página

1 O sólido de cores de Munsell . . . . . . . . . . . . . . . . 4

2 O disco de cores proposto por Munsell, com eixos cartesianos $a^{*}$ e $b^{*}$ adequadas para se avaliar uma cor segundo o sistema CIELAB . . . . . 5

3 Representação gráfica sobre um ponto da circunferência, dado pelo vetor e e o seu respectivo ângulo azimutal $\phi$ (a) ou ângulo polar $\theta$ (b) . . . 8

4 Componentes retangulares do vetor unitário e que representam o dado angular $\theta \ldots \ldots \ldots \ldots \ldots \ldots \ldots \ldots \ldots \ldots \ldots \ldots \ldots \ldots \ldots \ldots \ldots \ldots$

5 Cogumelos Pleurotus sajor-caju frescos utilizados no experimento de Moda (2003) . . . . . . . . . . . . . . . . . . . 14

6 Ângulo médio $\bar{x}_{0}$ de uma amostra de dados circulares $\theta_{1}, \theta_{2}, \ldots, \theta_{n}$ e vetor resultante correspondente de comprimento $\bar{R} \ldots \ldots$. . . . . 19

7 Funções densidade de probabilidade von Mises com $\mu=\frac{\pi}{4}$ e parâmetros de concentração $\kappa=1$ (vermelho), $\kappa=3$ (azul) e $\kappa=9$ (verde), na reta real (à esquerda) e na circuferência (à direita) . . . . . . . . . . 22

8 Gráfico da função $\hat{\kappa}=A_{1}^{-1}(\bar{R})$, para $0<\bar{R} \leq 0,9 \ldots \ldots \ldots 25$

9 Gráfico de dispersão angular para os três tratamentos simulados, respectivamente, com a indicação do vetor de comprimento igual ao raio médio, ângulo médio, em azul, média aritmética dos ângulos em vermelho . . . . 38

10 Gráfico quantil-quantil para os dados de tonalidade de cor simulados, apresentados na Tabela $1 \ldots \ldots \ldots$. . . . . . . . . . . 39 
11 Histogramas e densidades não-paramétricas para os dados de tonalidade de cor, em radianos, referentes aos três tratamentos simulados, respectivamente . . . . . . . . . . . . . . . . . . . . . . . 44 41 


\section{LISTA DE TABELAS}

Página

1 Dados angulares, em graus, simulados para um ensaio inteiramente ao acaso, com três tratamentos e 20 repetições, segundo distribuições de von Mises com médias $\mu_{1}=0^{0}, \mu_{2}=10^{0}$ e $\mu_{3}=355^{0}$ e $\kappa=100 \ldots \ldots$

2 Dados de cor, segundo o sistema CIELAB e de tonalidade, em graus, de cogumelos Pleurotus sajor-caju submetidos a quatro diferentes tratamentos (Trat: 1. Testemunha, 2. Peróxido de hidrogênio, 3. Água destilada, 4. Ácido cítrico.), logo após a aplicação e dez dias após a aplicação . . . 16

3 Resultados de um ensaio inteiramente ao acaso com $q$ tratamentos e $n_{j}$ $(j=1, \ldots, q)$ repetiçōes por tratamento $\ldots \ldots . \ldots . \ldots 27$

4 Análise de variância para o teste da hipótese $H_{0}: \mu_{1}=\cdots=\mu_{q}$, supondo $\tilde{\kappa} \geq 2\left(\right.$ ou $\left.\bar{R}^{\prime} \geq 0,45\right) \ldots \ldots \ldots 29$

5 Resultados do teste de comparações múltiplas para os dados da Tabela $3 \quad 32$

6 Média aritmética $\left(\bar{\theta}_{j}\right)$, ângulo médio $\left(\hat{\mu}_{j}\right)$, raio médio $\left(\bar{R}_{j}\right)$ e estimativa do parâmetro de concentração $\left(\hat{\kappa}_{j}\right)$ para os dados simulados de tonalidade de cor apresentados na Tabela 1. . . . . . . . . . . . 37

7 Quadro da análise de variância para os dados da Tabela 1, dados simulados, quando o método proposto for o de Stephens (1972) para dados

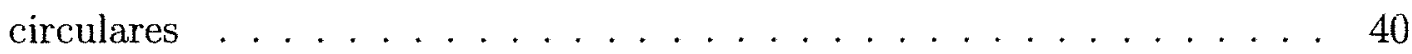

8 Quadro da análise de variância para os dados da Tabela 1, dados simulados, em graus, quando se utiliza o método usual . . . . . . . . . 40

9 Resultados do teste de comparações múltiplas aplicados aos dados da

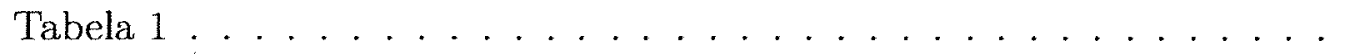


10 Média aritmética $\left(\bar{\theta}_{j}\right)$, ângulo médio $\left(\hat{\mu}_{j}\right)$, raio médio $\left(\vec{R}_{j}\right)$ e estimativa do parâmetro de concentração $\left(\hat{\kappa}_{j}\right)$ para dados de tonalidade de cor de cogumelo no tempo zero, apresentados na Tabela $2 \ldots \ldots 42$

11 Média aritmética $\left(\bar{\theta}_{j}\right)$, ângulo médio $\left(\hat{\mu}_{j}\right)$, raio médio $\left(\bar{R}_{j}\right)$ e estimativa do parâmetro de concentração $\left(\hat{\kappa}_{j}\right)$ para dados de tonalidade de cor de cogumelo no tempo dez, apresentados na Tabela $2 \ldots . . . . . . . .443$

12 Quadro da análise de variância para os dados de tonalidade de cor de cogumelo no tempo zero, apresentados na Tabela 2 , usando o método proposto por Stephens (1972) para dados circulares . . . . . . . . . . . 43

13 Quadro da análise de variância para os dados de tonalidade de cor de cogumelo no tempo dez, apresentados na Tabela 2, usando o método proposto por Stephens (1972) para dados circulares . . . . . . . . . . . . 44

14 Resultados do teste de comparações múltiplas aplicados aos dados da Tabela $2 \ldots \ldots \ldots \ldots . \ldots \ldots 4 . \ldots \ldots$

15 Quadro da análise de variância para os dados de tonalidade de cor de cogumelo no tempo zero, em graus, apresentados na Tabela 2, usando o método tradicional . . . . . . . . . . . . . . . . . 45

16 Quadro da análise de variância para os dados de tonalidade de cor de cogumelo no tempo dez, em graus, apresentados na Tabela 2, usando o método tradicional . . . . . . . . . . . . . . . . 


\title{
ANÁLISE DE VARIÂNCIA PARA DADOS DE TONALIDADE DE COR: UM CASO DE DADOS CIRCULARES
}

\author{
Autor: ANTONIO WiLLIAMS MOITA \\ Orientador: Prof Dr SILVIO SANDOVAL ZOCCHI
}

\section{RESUMO}

Em experimentos na área de tecnologia de alimentos, e de pós-colheita para produtos hortícolas, a cor é considerada um dos aspectos mais importantes na aparência do alimento, pois acredita-se que se o alimento não tiver uma boa aparência, muito provavelmente o consumidor não avaliará os outros aspectos como o sabor e a textura. Um dos principais sistemas para medir cores é o sistema CIELCH. Neste sistema a cor é uma variável aleatória tridimensional formada pelas variáveis $L$, $\mathrm{C}$ e H, respectivamente, luminosidade, saturação e tonalidade da cor. Esta última é um ângulo, ou seja, uma variável circular, que comumente possui distribuição de von Mises sendo, no entanto, analisada como tendo distribuição normal. Considerando-se os dados de tonalidade relativos de um experimento inteiramente ao acaso, este trabalho sugere como alternativa, a utilização da metodologia de análise de variância 
para dados circulares proposta por Watson \& Williams (1956) e modificada por Stephens (1972). Propõe-se, ainda, um método de comparações múltiplas para as médias angulares relativos aos tratamentos, baseado na estatística de Watson \& Williams (1956) e Stephens (1972). Como forma empírica de se verificar a pressuposição de que os dados provém de distribuição de von Mises com parâmetros de concentração iguais, propõe-se a construção de um gráfico quantil-quantil. Como forma de ilustrar a metodologia, foram analisados dois conjuntos de dados, sendo o primeiro, relativo a um experimento inteiramente ao acaso com três tratamentos, extraídos de distribuições de von Mises com médias $0^{0}, 10^{\circ}$ e $355^{\circ}$, ou seja, todos com a tonalidade de cor vermelha, e parâmetros de concentração iguais a 100. O segundo conjunto de dados, por outro lado, provém de um experimento inteiramente ao acaso realizado por Moda (2003), na área de tecnologia de alimentos e, que teve como objetivo, avaliar a conservação de cogumelos Pleurotus sajor-caju frescos quanto à qualidade e à durabilidade, quando submetidos a quatro diferentes tratamentos usuais na conservação de alimentos. As análises destes dois conjuntos de dados, evidenciam os problemas graves que podem ocorrer quando se utiliza a metodologia proposta por McGuire (1992) que não ocorrem quando se utiliza a proposta nesse trabalho. 


\title{
ANALYSIS OF VARIANCE FOR DATA COLOR HUE: A CASE OF CIRCULAR DATA
}

\author{
Author: ANTONIO WILLIAMS MOITA \\ Adviser: Prof. Dr. SILVIO SANDOVAL ZOCCHI
}

\section{SUMMARY}

In experiments in the area of food technology and post-harvest for horticultural products, the color is considered one of the most important aspects in the food appearance, since it is believed that if the food does not have a good appearance, the consumer certain will not evaluate the other aspects of flavour and texture. One of the main systems to measure colors is the CIELCH. In this system the color is a three-dimensional random variable formed by variables $\mathrm{L}, \mathrm{C}$ e $\mathrm{H}$, respectively, lightness, chroma and hue. The later is an angle, e.g. a circular variable, that usually has a von Mises distribution but is, analysed as having a normal distribution. Considering color hue data relative to a completely randomized experiment, this work suggests, as an alternative, the use of analysis of variance methodology for circular data, as proposed by Watson \& Williams (1956) and modified by Stephens (1972). A multiple comparisons method to the angles means relative for the treatments, based on the statistics of Watson \& Williams (1956) and Stephens (1972), 
is also proposed. As an empirical way of verify the assumption that the data come from von Mises distribution with equal concentration parameters, the construction of a quantile-quantile plot is proposed. To ilustrate the methodology, two data sets were analysed. The former from a completely randomized experiment with three treatments, extracted from a von Mises distribution with means equal to $0^{0}, 10^{\circ}$ and $355^{\circ}$, e.g., all with red color hue, and concentration parameters equal to 100 . The second data set, on the other hand, comes from a completely randomized experiment carried out by Moda (2003), in food technology and that had as objective the evaluation of the conservation of fresh mushroom Pleurotus sajor-caju in the sense of quality and durability, when submitted to four different usual treatments in the food conservation. These two data sets analyses demonstrated the serious problems that can occur if the methodology proposed by McGuire (1992) is used, but these problems do not occur if one uses the methods proposed here. 


\section{INTRODUÇÃO}

Dados direcionais, de uma maneira geral, podem ser vistos como pontos sobre uma hiperesfera com raio igual a um, visualizados em um espaço de qualquer dimensão. Contudo, na prática, quase que invariavelmente situam-se no espaço de duas ou três dimensões. Quando estes ocorrem no espaço tridimensional são denominados dados esféricos e, no bidimensional, dados circulares. Dados direcionais são ângulos e variam no intervalo de $0^{0}$ a $360^{\circ}$ ou de 0 a $2 \pi$ radianos. Portanto, dados circulares podem ser vistos como pontos sobre uma circunferência de raio unitário.

A análise de dados circulares tem aplicações em muitas áreas, como: Biologia (navegação e orientação dos animais), Meteorologia (direção dos ventos), Astronomia, Física, Geologia (paleomagnetismo), Medicina, Odontologia etc. Tem aplicação ainda, em qualquer área em que haja interesse em avaliar as cores quando medidas no sistema CIELCH, que é um dos padrões de cor definido pela Comissão Internacional de Iluminação. Segundo esse sistema, a cor é uma variável aleatória tridimensional formada pelas variáveis $\mathrm{L}, \mathrm{C}$ e $\mathrm{H}$, sendo $\mathrm{L}$, a luminosidade, $\mathrm{C}$, a saturação e H, a tonalidade da cor, que é um ângulo medido em radianos ou graus.

$\mathrm{Na}$ área de tecnologia de alimentos, inúmeros estudos têm contribuído para a análise e o desenvolvimento de pesquisas na área de pós-colheita de produtos hortícolas e na indústria de alimentos. Nesses estudos, a cor é considerada um dos aspectos mais importantes da aparência do alimento, pois acredita-se que se o alimento não tiver uma boa aparência, muito provavelmente o consumidor não será atraído por ela e deixará de avaliar outros aspectos do alimento como o sabor e a textura. Dentre as variáveis que compōem a cor, a tonalidade é citada como uma 
das principais para a avaliação de cor de alimentos.

Apesar de a tonalidade da cor ser uma medida angular, expressa em graus ou radianos, em diversos artigos científicos, a análise desse aspecto é feita como se fosse uma medida pertencente à reta real, o que pode levar a conclusões incorretas. Esses problemas poderiam, no entanto, ser evitados, utilizando-se métodos estatísticos apropriados, ou seja, que levam em conta a periodicidade do círculo nos seus métodos de estimação.

Nesse trabalho, faz-se uma revisão sobre a metodologia para análise de variância para dados circulares, apresentando o método proposto por Stephens (1972), com ênfase na análise de dados da tonalidade de cor. Essa metodologia simples poderá, desse modo, contribuir para solucionar as dificuldades hoje presentes nas análises estatísticas das pesquisas de pós-colheita que envolvem a tonalidade da cor dos alimentos. Além disso, poderá ser utilizada em estudos de ecologia, entomologia e geologia que envolvem análise de variância para dados circulares.

Faz-se, ainda, um estudo da metodologia sugerida por McGuire (1992) para a análise de dados de tonalidade da cor de frutas e hortaliças, que considera a tonalidade como se fosse uma medida pertencente à reta real e tendo distribuição normal

Como forma de ilustrar a aplicação dessas metodologias, são analisados dois conjuntos de dados, sendo o primeiro, obtido por simulação e o segundo, referente a um ensaio comparativo de diferentes tratamentos para a conservação do cogumelo Pleurotus sajor-caju, obtidos por Moda (2003). 


\section{REVISÃO DE LITERATURA}

A aparência, o sabor e a textura são considerados os três principais aspectos na avaliação dos alimentos, sendo a aparência, o mais importante por ser a primeira e a mais simples das avaliações, pois acredita-se que se o alimento não tiver uma boa aparência, muito provavelmente o consumidor não avaliará os outros dois aspectos do alimento: sabor e textura. Dentre os aspectos da aparência: cor, brilho, luminosidade e estado físico, a cor, é considerada por muitos estudiosos, o aspecto mais importante na aparência do alimento (Clydesdale, 1993; Francis, 1980, 1998a).

A avaliação ou descrição das cores pode ser feita com a utilização de designações verbais, numéricas, ou em termos matemáticos, como utilizado na instrumentação. A classificação das cores é um processo que se iniciou com a estimação visual e a construção de atlas de cores (Francis, 1998a).

O mais importante atlas de cores é conhecido como "Sistema Munsell" e foi concebido por Albert Henry Munsell, artista plástico americano, que iniciou a construção do seu modelo em 1890. Em 1905, propôs o sistema que leva o seu nome e é um dos padrões do US National Bureau of Standards e do CIE (Comissão Internacional de lluminação), além de ser também, o padrão mais utilizado na ciência, na indústria, nas artes e na colorimetria (medição da cor).

Munsell desejava com esse sistema, criar uma "maneira racional para classificar as cores", pois considerava "ridícula" e "ilusória" a classificação por nomes. Para isso, idealizou um sistema de classificação numérica em que cada cor tem um local próprio em um sólido de cores representado pela Figura 1.

Esse sistema é formado por um sólido cuja altura reflete a luminosidade 


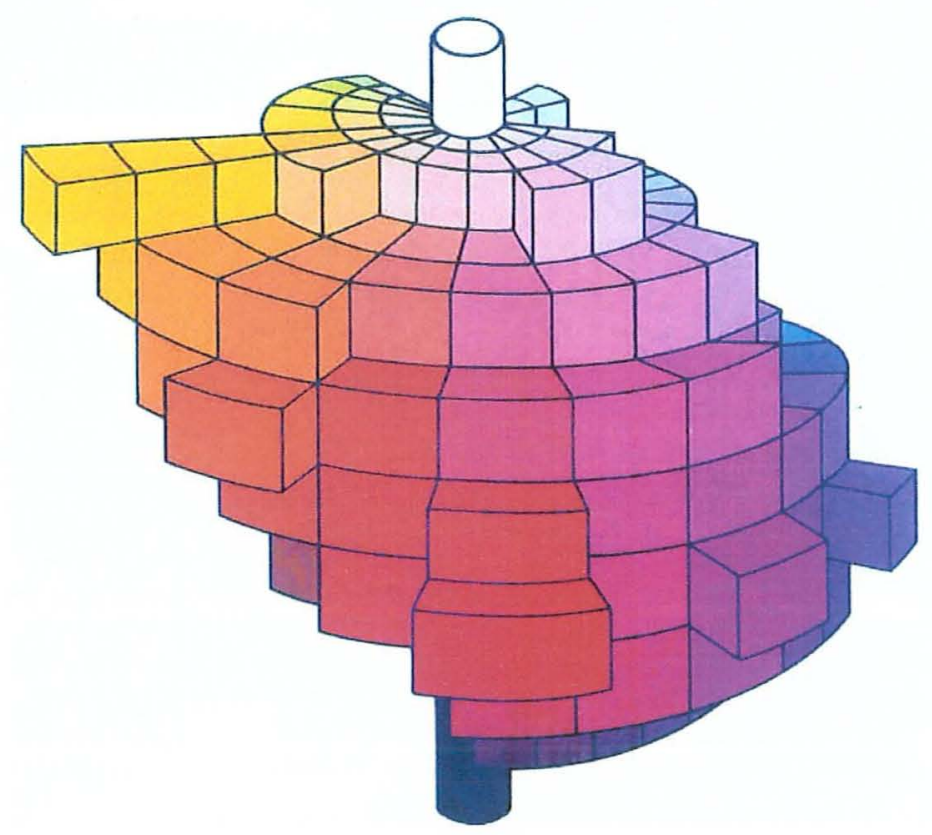

Figura 1 - O sólido de cores de Munsell

da cor e cujos cortes em certas alturas, formam discos que contém as cores, e apresentam uma gradação do cinza neutro, no centro, até a saturação na circunferência, conforme ilustra a Figura 2.

Para localizar uma determinada cor no seu sólido de cores, Munsell definiu três variáveis: "Hue" ou tonalidade, "Value" ou luminosidade e "Chroma" ou intensidade.

A tonalidade, ou nuance é a forma como se distingue uma cor de uma outra, e é formada por cinco cores primárias: vermelho, amarelo, verde, azul e púrpura e cinco cores intermediárias: amarelo-vermelho, verde-amarelo, azul-verde, púrpura-azul e vermelho-púrpura que, para efeito de classificação, estão arranjadas em um disco dividido em 100 partes iguais.

A luminosidade, por sua vez, é definida como o critério pelo qual se distingue uma cor clara de uma cor escura. No sistema originalmente proposto, a luminosidade variava de $1 \mathrm{~N}$ (preto) a $9 \mathrm{~N}$ (branco), posteriormente modificada para 


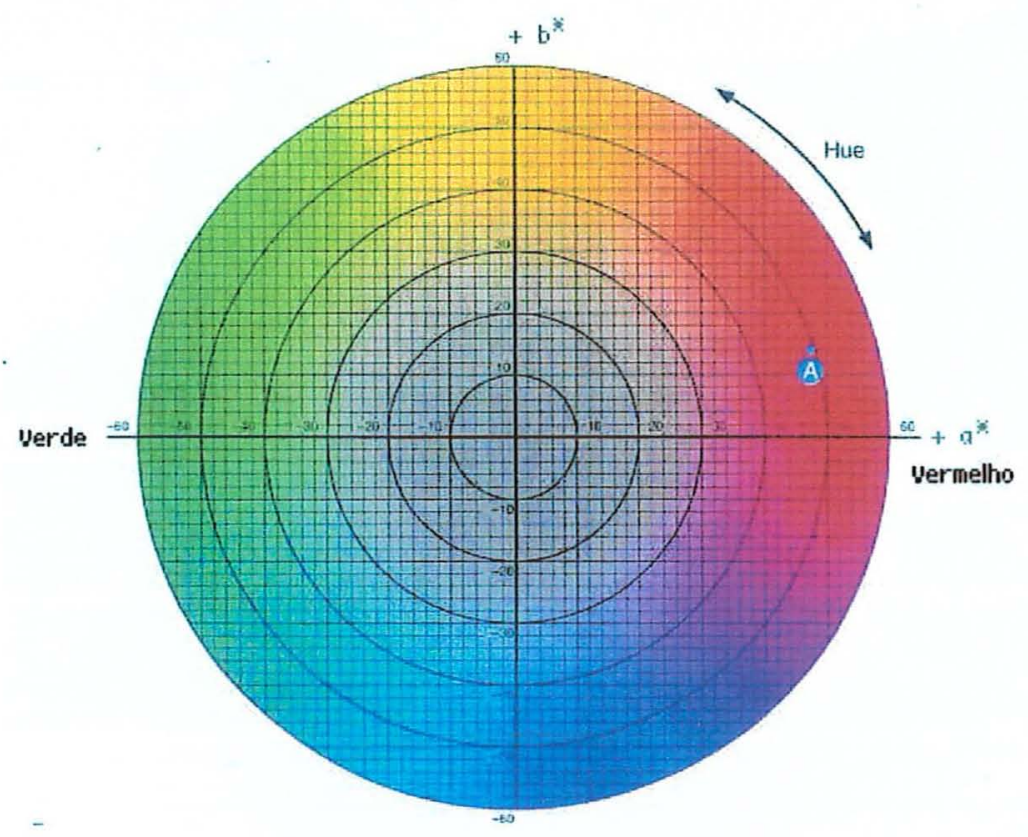

Figura 2 - O disco de cores proposto por Munsell, com eixos cartesianos $a^{*}$ e $b^{*}$ adequadas para se avaliar uma cor segundo o sistema CIELAB

0 (preto) a 10 (branco).

A intensidade, refere-se à propriedade de distinguir as diferenças entre uma tonalidade pura e gradações do cinza.

O Atlas Munsell de Cores contém 1225 retângulos de cores, cada um deles designado por um número, cujas medidas variam, normalmente, de $\frac{5}{8}$ por $\frac{7}{8}$ polegadas até $8 \frac{1}{2}$ por $11 \frac{1}{2}$ polegadas. Como cada cor tem uma localização diferente no sólido de cores, quando uma cor é especificada pela designação de Munsell, ela não é ambígua.

Assim, tabelas distintas de cores foram criadas para particularizar cores relativas a cabelo, tintas, solo, têxteis etc. Na área de alimentos, há padrões confeccionados em plástico para feijão, ervilha, suco de laranja, pêssego, salmão etc. Para açúcar, devido à fragilidade e ao esmaecimento da cor com o uso, foram criados 
padrões confeccionados em vidro (Francis, 1998a).

Apesar de ampla utilização de padrões de cores, este método vem sendo substituído, nos últimos anos, por métodos instrumentais.

Inicialmente, os métodos instrumentais para medir cores fundamentavam-se nos estudos das respostas do cone do olho humano em termos de espectro visível, desenvolvidos por fisiologistas. O experimento para a determinação de uma cor consistia em colocar filtros vermelho, verde e azul na frente das lentes de três projetores, e projetar as três luzes sobre a metade de um círculo, e na outra metade, projetar uma cor desconhecida. Se a quantidade de luz proveniente de cada um dos projetores pudesse ser controlada, então um observador poderia regular a quantidade de luz vermelha, verde e azul necessárias para reproduzir a cor espectral desconhecida. A estas quantidades de vermelho, verde e azul que definem a cor espectral dá-se o nome de triestímulos (Francis, 1980, 1998a).

A partir dessa idéia, foram desenvolvidos aparelhos para medir a cor, chamados colorímetros de triestímulos. Para a construção desses aparelhos são necessários uma fonte de luz, três filtros de vidro com espectro de transmitância que duplicam as curvas $\mathrm{X}, \mathrm{Y}$, e Z e uma fotocélula. Com esse arranjo pode-se ler o valor de $(X, Y, Z)$ que representa a cor da amostra. Todos os colorímetros baseiam-se nesse princípio, variando apenas os refinamentos na resposta da fotocélula, na estabilidade, na sensibilidade e na reprodutibilidade. Apesar de o conceito ser semelhante para todos os espectrofotômetros, eles não usam as mesmas unidades, pois utilizam diferentes combinações de filtros-fotocélulas bem como diferentes eixos no espaço de cores, ou mesmo diferentes sólidos de cores. Contudo, algumas padronizações estão sendo desenvolvidas e quatro sistemas de cor instrumental vêm ganhando popularidade: CIE-XYZ, com variáveis XYZ, Judd-Hunter LAB, com variáveis Lab, CIELAB, com variáveis $L^{*} a^{*} b^{*}$ e CIELCH, com variáveis $L^{*} C^{*} H^{*}$ (Francis, 1998a).

O sistema de cor CIELAB, bastante utilizado na área de tecnologia de alimentos, é muito semelhante ao sistema Munsell de cores, no qual o disco que 
contém as cores (ver Figura 2), está centrado em um sistema de eixos cartesianos $a^{*} b^{*}$, baseados no fato de que uma cor não pode ser ao mesmo tempo vermelho e verde, ou azul e amarelo, porque estas cores opõem-se umas às outras. O eixo das abscissas $a^{*}$ apresentam valores que variam de -60 , representando a cor verde, a +60 , representando a cor vermelha e o eixo das ordenadas $b^{*}$ apresenta valores que variam de -60 , repesentando a cor azul, a +60 , representando o amarelo. Assim, a origem do sistema $(0,0)$ representa uma cor neutra representada pelo cinza. A luminosidade da cor, por sua vez, é dada pelo valor de $L^{*}$ que pode variar de 0 (preto) a 100 (branco) (ADOBE SYSTEMS INCORPORATED, 2003).

Segundo Francis (1987) e McGuire (1992) após a obtenção dos dados com a utilização de colorímetros de triestímulos, muitos pesquisadores simplesmente publicam os dados do espaço $L^{*} a^{*} b^{*}$ em uma tabela, sem apresentar qualquer análise e em muitos casos, sem nenhuma manipulação dos mesmos, ou quando muito, realizam a análise de variância para $L^{*}, a^{*}, b^{*}$ separadamente. Segundo os autores, as análises para os valores $a^{*}$ e $b^{*}$ não são apropriadas pois $a^{*}$ e $b^{*}$ são difíceis de serem interpretadas separadamente.

Sugerem como alternativa que a localização do ponto $\left(a^{*}, b^{*}\right)$ no disco de cores seja feita por coordenadas polares $(\theta, r)$ sendo $\theta$ o ângulo cujo seno é $\frac{b^{*}}{60}$ e cujo cosseno é $\frac{a^{*}}{60}$, módulo $360^{0}$ e $R$, a distância do ponto $\left(a^{*}, b^{*}\right)$ à origem $(0,0)$. Os valores $\theta$ e $R$, que representam a tonalidade e a saturação da cor, poderão ser, então, analisados separadamente.

Segundo Mardia (1972), dados circulares são pontos sobre a circunferência de um círculo de raio unitário. Logo, considerando-se o disco de cores como tendo raio unitário, e como a tonalidade são pontos sobre a circunferência deste disco, a tonalidade é um dado circular,

Dados circulares pertencem a uma classe de dados denominados de dados direcionais, que Mardia (1988) definiu matematicamente como um vetor aleatório $\mathbf{X}$ de dimensão $p\left(\mathbf{X} \in R^{p}\right)$ tal que $\mathbf{X}^{T} \mathbf{X}=1$, isto é, $\mathbf{X}$ encontra-se em uma esfera de dimensão $p-1$. 
Dados que representam direções podem ser tratados em um espaço de qualquer dimensão, mas em situações práticas, o interesse, geralmente, recai sobre o espaço de duas e três dimensões, e são representados graficamente por pontos sobre a superfície da circunferência de um círculo ou sobre a esfera. Em espaços de dimensão superior a três, as dimensões são representadas por pontos sobre a superfície de uma hiperesfera.

Dados circulares são representados graficamente por pontos sobre a circunferência de um círculo, centrada em um sistema de eixos cartesianos $x y$, cujo raio é igual a 1 (ver Figura 3(b)). Cada ponto pode ser representado por um vetor unitário e, cuja origem é o centro da circuferência e a extremidade final é o ponto sobre a circunferência do círculo de raio unitário. Pode ser representado, ainda, pelo ângulo $\theta\left(0^{0}<\theta \leq 360^{\circ}\right)$ formado pelo vetor unitário e e o vetor direção zero, em sentido contrário ao dos ponteiros do relógio, denominado de ângulo polar. No desenvolvimento da teoria de dados circulares e na maioria das aplicações, utiliza-se o eixo positivo de $x$ como sendo a direção zero (ver Figura 3(b)).

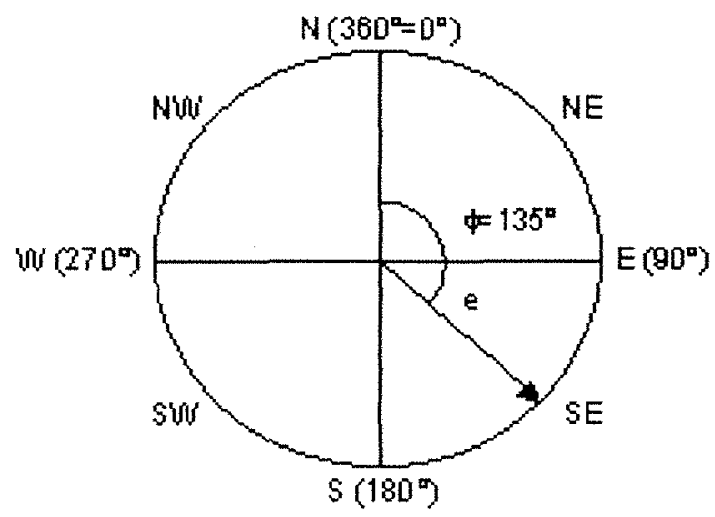

(a)

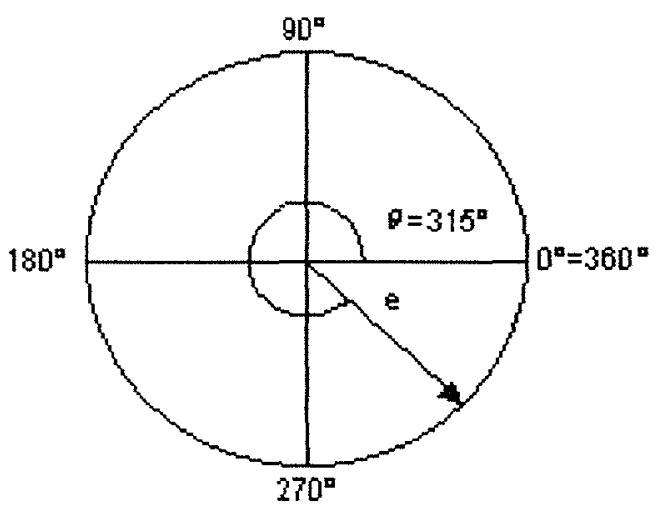

(b)

Figura 3 - Representação gráfica sobre um ponto da circunferência, dado pelo vetor e e o seu respectivo ângulo azimutal $\phi$ (a) ou ângulo polar $\theta(b)$

Dados circulares podem ser, também, medidos como ângulos azimutais. O ângulo azimutal $\phi$ é formado pelo vetor da direção zero, fixado como sendo o norte 
geográfico, e o vetor unitário e, no sentido dos ponteiros do relógio. Dessa forma, direção zero corresponde ao norte geográfico e a $0^{0}$, a direção leste a $90^{\circ}$, a direção sul a $180^{\circ}$, e a direção oeste a $270^{\circ}$ (ver Figura 3(a)). Os ângulo azimutais, assim definidos, são utilizados basicamente no estudo da direção dos ventos, orientação de animais, atividades exploratórias etc.

O ponto sobre a circunferência circular pode ser também representado em termos de coordenada cartesiana $(x, y)$, projetando-se o ponto sobre o eixo $x$ e sobre o eixo $y$, resultando na coordenada cartesiana do vetor $(\cos \theta, \operatorname{sen} \theta)$, enquanto as coordenadas polares são $(1, \theta)$ (ver Figura 4).

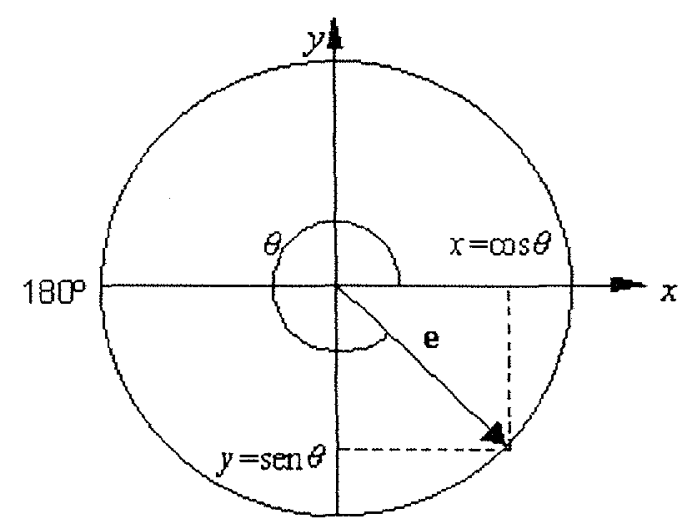

Figura 4 - Componentes retangulares do vetor unitário e que representam o dado angular $\theta$

Embora dados circulares sejam ângulos e, como tal, podem ser expressos em graus ou radianos, Mardia (1972) aconselha o uso de radianos, com intervalo de variação $0<\theta \leq 2 \pi$ ou $-\pi<\theta \leq \pi$, dependendo do interesse na análise.

Outro caso de dados circulares são os dados axiais, cujo interesse está na orientação de linhas sem direção, dos quais o estudo de fraturas de rochas é um exemplo. Na representação de dados axiais sobre uma hiperesfera, as linhas sem direção são estendidas para cortar a hiperesfera, e nenhuma distinção é feita entre os pontos diametralmente opostos. No caso de dados axiais sobre a circunferência do 
círculo de raio unitário, os ângulos $\theta\left(0<\theta \leq 180^{\circ}\right)$ e $\theta+180^{\circ}$ não são distinguíveis.

No estudo de dados direcionais, deve-se levar em conta as diferenças apresentadas na teoria estatística para o círculo e para a reta. O círculo é descrito como uma curva fechada enquanto que a reta não, portanto, as funções de distribuições, as funções características e os momentos devem levar em conta a periodicidade natural do círculo. Como exemplo dessa diferença, pode-se citar o caso da média aritmética de dois ângulos: $1^{0}$ e $359^{\circ}$ resultando em $180^{\circ}$, enquanto que, pela intuição geométrica, a média angular é $0^{0}$. Portanto, dados circulares como a direção do vento, o ângulo de fuga da migração das aves e medidas de tempo reduzidas a módulo de algum período e convertidas em ângulos, não podem ser tratados como dados lineares.

De acordo com Batschelet (1981), frequências diárias e sazonais "métodos utilizados para mensurações de ritmos biológicos" têm muito em comum com as distribuições circulares, pois podem-se converter em ângulos, medidas de tempo reduzidas a módulo de algum período de tempo. Nesse caso, os períodos de tempo mais utilizados são o dia e o ano e o comprimento do período de tempo é identificado com $360^{\circ}$ ou $2 \pi$. Mardia $(1972$, p.7) detalha essa conversão de tempo em ângulo e, considerando o período de tempo como ano, apresenta os fatores para a correção quando o mês de fevereiro tiver 28 ou 29 dias, bem como para os meses que têm 31 dias. Portanto, métodos estatísticos utilizados para ritmos biológicos são muito semelhantes aos aplicados a dados circulares. Contudo, há problemas devido ao fato de os dados serem geralmente coletados serialmente, conduzindo a pontos amostrais dependentes e o fato de que nem sempre o período exato é conhecido antecipadamente. Batschelet (1981) esclarece que, em tais condições, são requeridos métodos sofisticados de análise de séries temporais e recomenda tratar problemas de ritmos biológicos como dados circulares somente quando o comprimento do período for conhecido antecipadamente.

Segundo Batschelet (1981) e Zar (1999), a análise de dados circulares tem aplicações na Biologia, em estudos referentes à orientação e à navegação dos 
animais, ligados a atividades como a migração, retorno ao lar, fuga ou exploração; na Metereologia, a direção dos ventos; na Geologia, paleomagnetismo; na Astronomia, Física, Medicina, Odontologia etc.

Para se analisarem dados circulares, foram propostas inúmeras distribuições, sendo que, segundo Mardia (1972), a mais importante distribuição circular foi introduzida por von Mises em 1918. Essa distribuição, batizada pelo seu nome, foi criada quando pesquisava se os pesos atômicos eram números inteiros sujeitos a erros, isto é, a parte fracionária dos pesos atômicos era transformada em desvios angulares. Por exemplo, se a parte fracionária correspondia a $0,25=\frac{1}{4}$ então o seu desvio angular correspondente seria de $360^{\circ} \frac{1}{4}=90^{\circ}$; ou se a parte fracionária correspondia a $0,75=\frac{3}{4}$ então o seu desvio angular correspondente seria de $360^{\circ} \frac{3}{4}=270^{\circ}$, e assim por diante.

A inferência estatística direcional, segundo Harrison et al. (1986), foi iniciada com o trabalho de R. A. Fisher $(1953)^{1}$ relativo a distribuição sobre a esfera quando estudava o paleomagnetismo e auxiliou Greenwood \& Durand $(1955)^{2}$ a desenvolverem a teoria da distribuição circular.

No tocante à análise de variância para dados circulares os primeiros a desenvolverem e apresentarem os fundamentos teóricos necessários que unificaram os procedimentos de inferência para as distribuições de von Mises e Fisher foram Watson \& Williams (1956).

Dada uma amostra de uma distribuição de von Mises com parâmetro $\kappa$ desconhecido, um teste exato e outro aproximado para a hipótese de igualdade do vetor médio conhecido foi apresentado por Stephens (1962a e 1962b).

Posteriomente, Stephens (1972) elaborou um teste exato para a igualdade das direções médias, dadas duas amostras de distribuições de von Mises com iguais parâmetros de concentração $\kappa$, desconhecidos.

${ }^{1}$ FISHER, R.A. Dispersion on a sphere. Procedings of the Royal Society, London, Series A, 217, p.295-305, 1953

${ }^{2}$ GREENWOOD, J.A; DURAND, D. The distribuition of lenght and components of the sum of n random unit refers. Annals of Mathematical Statistics, v.26, p.233-246, 1955 
Usando os métodos de razão de verossimilhança, Upton (1974) desenvolveu testes de significância para ambas as situações: uma amostra e duas amostras.

O método de Watson \& Williams foi então estendido por Stephens (1982) para a análise hierárquica com dois fatores sem discutir, no entanto, a possibilidade do efeito da interação.

Seguindo um enfoque ligeiramente diferente de Stephens, Harrison et al. (1986) desenvolveram um método para delineamento em blocos ao acaso e de classificação dupla incluindo o efeito da interação.

Como forma de se testar se um conjunto de dados provém de uma distribuição de von Mises com parâmetros $\mu$ e $\kappa$, pressuposição básica para vários métodos relacionados até aqui, Lockhart \& Stephens (1985) propuseram um teste formal.

Um programa para a conversão de dados de cores no sistema CIELAB para o sistema CIELCH foi apresentado por McGuire (1992), como sendo o mais adequado à análise de cores de alimentos. Em seguida, desconsiderando o fato de os dados de tonalidade de cor serem dados angulares, esse autor propôs para o teste da hipótese de igualdade das médias aritméticas populacionais das tonalidades, a utilização de análise de variância e a utilização de métodos de comparações múltiplas usuais. Seguindo essa idéia, inúmeros pesquisadores da área de tecnologia de alimentos, como D'Souza et al. (1992) e Bible \& Singha (1993) analisaram dados angulares como não angulares. 


\section{MATERIAL}

O primeiro conjunto de dados, apresentado na Tabela 1 , tem a função de ressaltar os problemas que podem surgir quando se analisam dados circulares como pertencentes à reta real. Tratam-se de dados simulados de distribuições de von Mises com parâmetro de concentração $\kappa=100$ e três diferentes médias, $\mu_{1}=0^{0}$, $\mu_{2}=10^{0}$ e $\mu_{3}=355^{0}$, representando três tratamentos com 20 repetições cada, em um ensaio inteiramente ao acaso. Esses dados foram simulados utilizando-se o pacote estatístico R (Venables, 2000), e o pacote de funções CircStats (Agostinelli, 2002).

O segundo conjunto de dados, apresentado na Tabela 2, provém do experimento realizado por Moda (2003), na área de Tecnologia de Alimentos, com o objetivo de avaliar a conservação de cogumelos Pleurotus sajor-caju frescos quanto à qualidade e à durabilidade, quando submetidos a diferentes tratamentos usuais para a conservação de cogumelos.

Os cogumelos analisados foram produzidos no laboratório do Departamento de Agroindústria, Alimentos e Nutrição da Escola Superior de Agricultura "Luiz de Queiroz" e a análise referente à conservação foi feita no laboratório do Departamento de Entomologia e Irradiação de Alimentos do Centro de Energia Nuclear na Agricultura - CENA, localizados no campus "Luiz de Queiroz" da Universidade de São Paulo em Piracicaba, SP.

O delineamento experimental utilizado foi o inteiramente ao acaso com quatro tratamentos e cinco repetições, sendo a unidade experimental uma bandeja de

poliestireno com $200 \mathrm{~g}$ de cogumelo, embalada com filme plástico após o tratamento. Um tratamento foi considerado como testemunha, ou seja, os cogumelos não foram imersos em nenhum tipo de solução. Os outros três correspondem, respectivamente, 
a imersões em solução de peróxido de hidrogênio, água destilada pura e solução de ácido cítrico.

Foram efetuadas mensurações em três épocas distintas, logo após a aplicação dos tratamentos (tempo zero), no quinto e no décimo dia subseqüentes, sendo que, para efeito deste trabalho são considerados apenas os dados coletados no tempo zero e no décimo dia.

Para a avaliação da cor, utilizou-se o colorímetro de triestímulos Minolta CR-200 b de $8 \mathrm{~mm}$ de diâmetro, que utiliza o sistema CIELAB, ou seja, apresenta as cores através dos valores $L^{*}, a^{*}$ e $b^{*}$. O aparelho foi previamente calibrado em superfície branca de acordo com a Comissão Internacional de Iluminação (CIE) e com padrões pré-estabelecidos segundo Bible \& Singha (1993).

A Figura (5) apresenta uma foto dos cogumelos utilizados no experimento de Moda (2003).

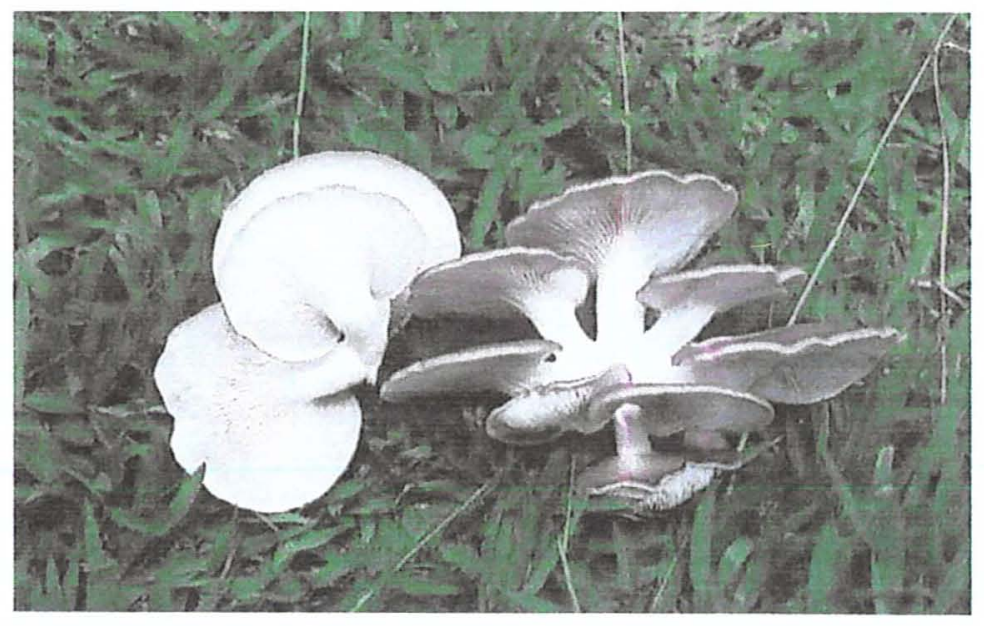

Figura 5 - Cogumelos Pleurotus sajor-caju frescos utilizados no experimento de Moda (2003) 
Tabela 1. Dados angulares, em graus, simulados para um ensaio inteiramente ao acaso, com três tratamentos e 20 repetições, segundo distribuições de von Mises com médias $\mu_{1}=0^{0}, \mu_{2}=10^{0}$ e $\mu_{3}=355^{0}$ e $\kappa=100$

\begin{tabular}{rrrr}
\hline \multicolumn{4}{c}{ Tratamentos } \\
Repetição & \multicolumn{1}{c}{1} & \multicolumn{1}{c}{2} & \multicolumn{1}{c}{3} \\
\hline 1 & 359,57 & 9,05 & 344,06 \\
2 & 357,35 & 4,96 & 351,89 \\
3 & 357,94 & 5,80 & 354,80 \\
4 & 3,02 & 13,40 & 351,72 \\
5 & 4,50 & 358,87 & 0,71 \\
6 & 3,41 & 4,50 & 354,68 \\
7 & 0,72 & 13,14 & 349,49 \\
8 & 358,39 & 9,71 & 354,25 \\
9 & 357,85 & 9,96 & 351,88 \\
10 & 3,17 & 2,73 & 348,96 \\
11 & 9,52 & 13,13 & 358,35 \\
12 & 359,63 & 12,66 & 355,61 \\
13 & 356,69 & 5,74 & 357,79 \\
14 & 8,66 & 9,75 & 351,19 \\
15 & 5,40 & 353,81 & 343,34 \\
16 & 358,20 & 12,46 & 349,56 \\
17 & 354,63 & 10,07 & 351,90 \\
18 & 357,37 & 5,74 & 353,40 \\
19 & 349,73 & 5,25 & 345,71 \\
20 & 358,04 & 4,33 & 352,49 \\
\hline & & &
\end{tabular}


Tabela 2. Dados de cor, segundo o sistema CIELAB e de tonalidade, em graus, de cogumelos Pleurotus sajor-caju submetidos a quatro diferentes tratamentos (Trat: 1. Testemunha, 2. Peróxido de hidrogênio, 3. Água destilada, 4. Ácido cítrico.), logo após a aplicação e dez dias após a aplicação

\begin{tabular}{ccccccccc}
\hline \multicolumn{7}{c}{ Após a Aplicação } & \multicolumn{5}{c}{ Dez dias Após a Aplicação } \\
Trat & $a^{*}$ & $b^{*}$ & $L^{*}$ & Tonalidade & $a^{*}$ & $b^{*}$ & $L^{*}$ & Tonalidade \\
\hline 1 & & & & & & & & \\
1 & $-0,5000$ & 7,2667 & 79,1000 & 93,94 & 0,0000 & 18,5333 & 79,1333 & 90,00 \\
1 & $-0,7333$ & 7,2000 & 79,5667 & 95,82 & $-0,3333$ & 16,5667 & 78,7333 & 91,15 \\
1 & $-0,6667$ & 8,2000 & 86,4333 & 94,65 & 0,3000 & 17,8000 & 79,6000 & 89,03 \\
1 & $-0,6333$ & 6,7000 & 83,2333 & 95,40 & 0,0000 & 14,9000 & 79,9333 & 90,00 \\
& $-0,6667$ & 9,4667 & 83,9333 & 94,03 & $-0,0667$ & 15,3667 & 80,0000 & 90,25 \\
2 & $-1,0667$ & 6,8000 & 70,7333 & 98,92 & $-0,0333$ & 18,9667 & 70,7667 & 90,10 \\
2 & $-0,8667$ & 7,3000 & 64,8333 & 96,77 & 0,6667 & 18,7000 & 67,1000 & 87,96 \\
2 & $-1,3333$ & 8,2333 & 67,5333 & 99,20 & 0,2333 & 16,6000 & 71,6333 & 89,19 \\
2 & $-0,9333$ & 5,4667 & 65,8667 & 99,69 & $-0,8667$ & 15,2667 & 67,4000 & 93,25 \\
2 & $-1,1333$ & 7,5000 & 64,5667 & 98,59 & $-0,3000$ & 18,8000 & 74,4667 & 90,92 \\
& & & & & & & & \\
3 & $-1,0667$ & 7,9000 & 78,8000 & 97,69 & 0,1333 & 18,6000 & 74,9667 & 89,59 \\
3 & $-0,8000$ & 7,4000 & 72,3667 & 96,17 & $-0,4333$ & 17,6000 & 73,4667 & 91,41 \\
3 & $-0,8000$ & 6,5333 & 68,7333 & 96,98 & $-0,0333$ & 18,5667 & 70,4000 & 90,10 \\
3 & $-1,0667$ & 6,7667 & 75,1667 & 98,96 & 1,1333 & 15,4667 & 74,8000 & 94,19 \\
3 & $-0,7667$ & 6,1333 & 66,5333 & 97,13 & 0,4667 & 18,0000 & 71,6333 & 88,52 \\
4 & $-1,6000$ & 7,2333 & 70,8667 & 102,47 & $-1,4667$ & 15,1667 & 68,9333 & 95,52 \\
4 & $-1,6000$ & 9,1333 & 75,4000 & 99,94 & $-1,8333$ & 18,1000 & 71,5667 & 95,78 \\
4 & $-1,3333$ & 8,3667 & 75,3333 & 99,05 & 0,2667 & 19,3667 & 73,6333 & 89,21 \\
\hline & $-1,9667$ & 7,6667 & 72,7333 & 104,39 & $-1,8000$ & 14,7333 & 68,3333 & 96,97 \\
& $-1,3667$ & 7,4667 & 76,2667 & 100,37 & $-0,5000$ & 19,4000 & 71,5000 & 91,48 \\
\hline
\end{tabular}

Fonte: Moda (2003) 


\section{MÉTODOS}

Considere, inicialmente, que se tenha um conjunto de $n$ dados de cor obtidos segundo o sistema CIELAB, ou seja,

$$
\left\{\left(a_{1}^{*}, b_{1}^{*}, L_{1}^{*}\right),\left(a_{2}^{*}, b_{2}^{*}, L_{2}^{*}\right), \ldots,\left(a_{n}^{*}, b_{n}^{*}, L_{n}^{*}\right)\right\}
$$

cuja notação, sem perda de generalidades, será simplificada para

$$
\left\{\left(a_{1}, b_{1}, L_{1}\right),\left(a_{2}, b_{2}, L_{2}\right), \ldots,\left(a_{n}, b_{n}, L_{n}\right)\right\}
$$

De modo a se obterem os dados de tonalidade de cor, $\theta_{i}(i=1, \ldots, n)$, os pares de dados $\left(a_{1}, b_{1}\right), \ldots,\left(a_{n}, b_{n}\right)$ devem ser transformados em ângulos, sistema CIELCH, da seguinte forma

$$
\theta_{i}=\left\{\begin{array}{llll}
\operatorname{arctg} \frac{b_{i}}{a_{i}}, & \text { se } \quad b_{i} \geq 0 \text { e } & a_{i}>0 \\
\operatorname{arctg} \frac{b_{i}}{a_{i}}+\pi, & \text { se } & a_{i}<0 & \\
\operatorname{arctg} \frac{b_{i}}{a_{i}}+2 \pi, & \text { se } & b_{i}<0 \text { e } & a_{i}>0 \\
\frac{\pi}{2}, & \text { se } & b_{i}>0 \text { e } & a_{i}=0 \\
\frac{3 \pi}{2}, & \text { se } & b_{i}<0 \text { e } & a_{i}=0 \\
\text { indeterminado, }, & \text { se } & b_{i}=0 \text { e } & a_{i}=0
\end{array} .\right.
$$

Considere, agora, que se tenha um conjunto de $n$ dados circulares, $\left\{\theta_{1}, \theta_{2}, \ldots, \theta_{n}\right\}$. Como passo inicial para a análise dos mesmos, será visto, a seguir, como sumariá-los por meio de medidas de locação e de dispersão. Assim, sejam $\bar{S}$ e $\bar{C}$ as médias das projeções dos ângulos $\theta_{i}(i=1, \ldots, n)$, sobre os eixos das ordenadas 
e das abscissas, respectivamente, dadas por

$$
\bar{S}=\frac{1}{n} \sum_{1=1}^{n} \operatorname{sen} \theta_{i}
$$

e

$$
\bar{C}=\frac{1}{n} \sum_{i=1}^{n} \cos \theta_{i} .
$$

Seja, ainda, $\bar{x}_{0}$, tal que,

$$
\operatorname{tg} \bar{x}_{0}=\frac{\bar{S}}{\bar{C}}
$$

ou seja,

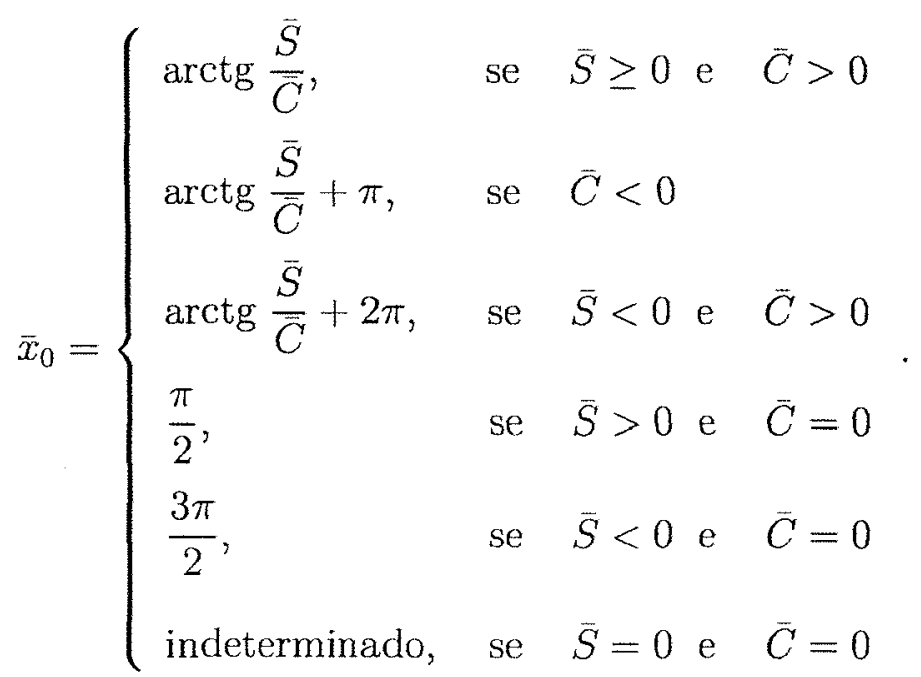

A medida definida pela eq. (3), chamada ângulo médio, é a mais importante medida de locação para dados circulares e pode ser visualizada na Figura 6.

Considere, agora, o vetor $[\bar{C}, \bar{S}]^{T}$, também representado na Figura 6 . Seu comprimento, dado por

$$
\bar{R}=\sqrt{\bar{S}^{2}+\bar{C}^{2}}
$$

pode assumir valores de 0 a 1 , e é uma medida comumente utilizada para se avaliar o grau de concentração dos pontos ao redor do ângulo médio $\bar{x}_{0}$. Como $\bar{R}$ é uma medida de concentração, $1-\bar{R}$ pode ser considerada uma medida de dispersão. Assim, a ausência de dispersão seria indicada por $1-\vec{R}=0$ e a máxima dispersão, por $1-\bar{R}=1$. Essa medida de dispersão, definida como variância circular por Mardia (1972), é dada por

$$
S^{2 *}=1-\bar{R}
$$




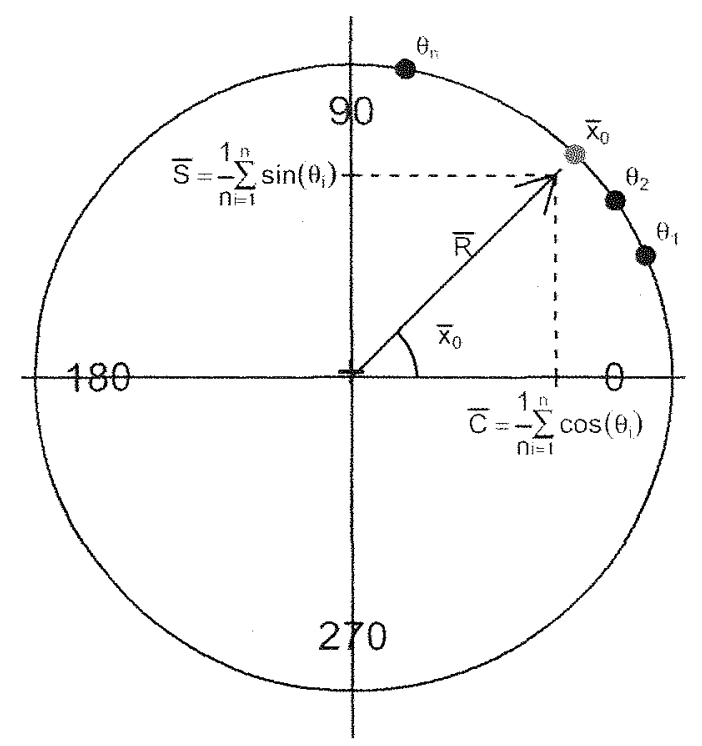

Figura 6 - Angulo médio $\bar{x}_{0}$ de uma amost ta de darlos cirenlares $\theta_{1}, \theta_{2} \ldots \theta_{3 i}$ e vetor resultante compespendente de comprimento $\bar{h}$

e é uma estatística que varia de 0 a 1.

Por outro lado, baseando-se no fato de que o desenvolvimento da função $\cos x$ em série de MacLaurin é dado por

$$
\cos x \approx 1-\frac{x^{2}}{2}
$$

isto é,

$$
2(1-\cos x) \approx x^{2}
$$

temos então que

$$
2 \sum_{i=1}^{n}\left(1-\cos x_{i}\right) \approx \sum_{i=1}^{n} x_{i}^{2}
$$

ou seja

$$
2 \sum_{i=1}^{n}\left(1-\cos \left(\theta_{i}-\bar{\theta}\right)\right) \approx \sum_{i=1}^{n}\left(\theta_{i}-\bar{\theta}\right)^{2}
$$

mas de (15) tem-se que

$$
\sum_{i=1}^{n} \cos \left(\theta_{i}-\bar{\theta}\right)=R
$$


$\log \mathrm{O}$

$$
2 n(1-\bar{R}) \approx \sum_{i=1}^{n}\left(\theta_{i}-\bar{\theta}\right)^{2}
$$

portanto temos que

$$
\frac{1}{n} \sum_{i=1}^{n}\left(\theta_{i}-\bar{\theta}\right)^{2} \approx 2(1-\bar{R})
$$

sendo

$$
\bar{\theta}=\frac{1}{n} \sum_{i=1}^{n} \theta_{i}
$$

Batschelet (1981) define variância angular como

$$
s^{2}=2 S^{2 *}=2(1-\bar{R})
$$

De modo a criar uma medida de dispersão que pudesse assumir valores dentro do campo dos reais positivos, Mardia (1972) define a chamada medida de variância como

$$
s_{0}^{2}=-2 \ln \bar{R}
$$

Convém observar que as medidas de dispersão dadas pelas equações (4), (5) e (6) são expressas em radianos ao quadrado e, caso haja o interesse em expressá-las em graus ao quadrado, deve-se multiplicá-las por $\left(180^{\circ} / \pi\right)^{2}$.

Com o objetivo de se fazer inferência, considere, agora, $\theta_{1}, \theta_{2}, \ldots, \theta_{n}$ como uma amostra aleatória de tamanho $n$ de uma variável aleatória $\Theta$. Supondo que a variável aleatória $\Theta$ tem distribuição de von Mises com parâmetros $\mu$ e $\kappa$, sua função densidade de probabilidade é dada por

$$
f_{\Theta}(\theta ; \mu, \kappa)=\frac{1}{2 \pi I_{0}(\kappa)} \exp [\kappa \cos (\theta-\mu)]
$$

sendo $0<\theta \leq 2 \pi, 0 \leq \mu<2 \pi, \kappa>0$ e $I_{0}(\kappa)$, uma função de Bessel modificada do primeiro tipo e de ordem zero, dada por:

$$
I_{0}(\kappa)=\sum_{r=0}^{\infty} \frac{1}{r !^{2}}\left(\frac{1}{2} \kappa\right)^{2 r}=1+\sum_{r=1}^{\infty} \frac{1}{r !^{2}}\left(\frac{1}{2} \kappa\right)^{2 r}
$$


Seja $I_{p}(\kappa)$ uma função de Bessel modificada do primeiro tipo de ordem $p$ definida pela série

$$
I_{p}(\kappa)=\sum_{r=0}^{\infty} \frac{1}{(p+r) ! r !}\left(\frac{1}{2} \kappa\right)^{2 r+p}
$$

cujo caso particular $p=0$ é a função (8). A função definida por

$$
A_{p}(\kappa)=\frac{I_{p}(\kappa)}{I_{0}(\kappa)}
$$

é utilizada para descrever algumas características da distribuição de von Mises, tais como o comprimento médio resultante

$$
\rho=A_{1}(\kappa)
$$

e a dispersão circular de $\Theta$

$$
\delta=\left[\kappa A_{1}(\kappa)\right]^{-1}
$$

dentre outras.

A distribução de von Mises possui, ainda, as seguintes propriedades: é unimodal, simétrica em torno do ângulo médio $\mu$, converge para a distribuição uniforme circular quando $\kappa$ tende a 0 , e converge para a distribuição degenerada no ponto $\theta=\mu$, quando $\kappa$ tende ao infinito.

Como ilustração, a Figura 7 apresenta gráficos de funções densidade de probabilidade von Mises com parâmetro de locação $\mu=\frac{\pi}{4}$ e parâmetros de concentração $\kappa=1, \kappa=3$ e $\kappa=9$ considerando-se a reta real e a circuferência.

A partir da equação (7), obtém-se a função de distribuição de $\Theta$, dada por:

$$
F_{\Theta}(\theta)=\frac{1}{2 \pi I_{0}(\kappa)} \int_{0}^{\theta} \exp [\kappa \cos (t-\mu)] d t
$$

para a qual não existe uma função primitiva.

Dada uma amostra aleatória $\theta_{1}, \ldots, \theta_{n}$ de uma variável aleatória $\Theta$ com distribuição von Mises com parâmetros $\mu \mathrm{e} \kappa$, a função de verossimilhança de $\mu$ e $\kappa$ é dada por

$$
L(\mu, \kappa)=\prod_{i=1}^{n} f_{\Theta}\left(\theta_{i} ; \mu, \kappa\right)=\frac{1}{\left[2 \pi I_{0}(\kappa)\right]^{n}} \exp \left[\sum_{i=1}^{n} \kappa \cos \left(\theta_{i}-\mu\right)\right]
$$




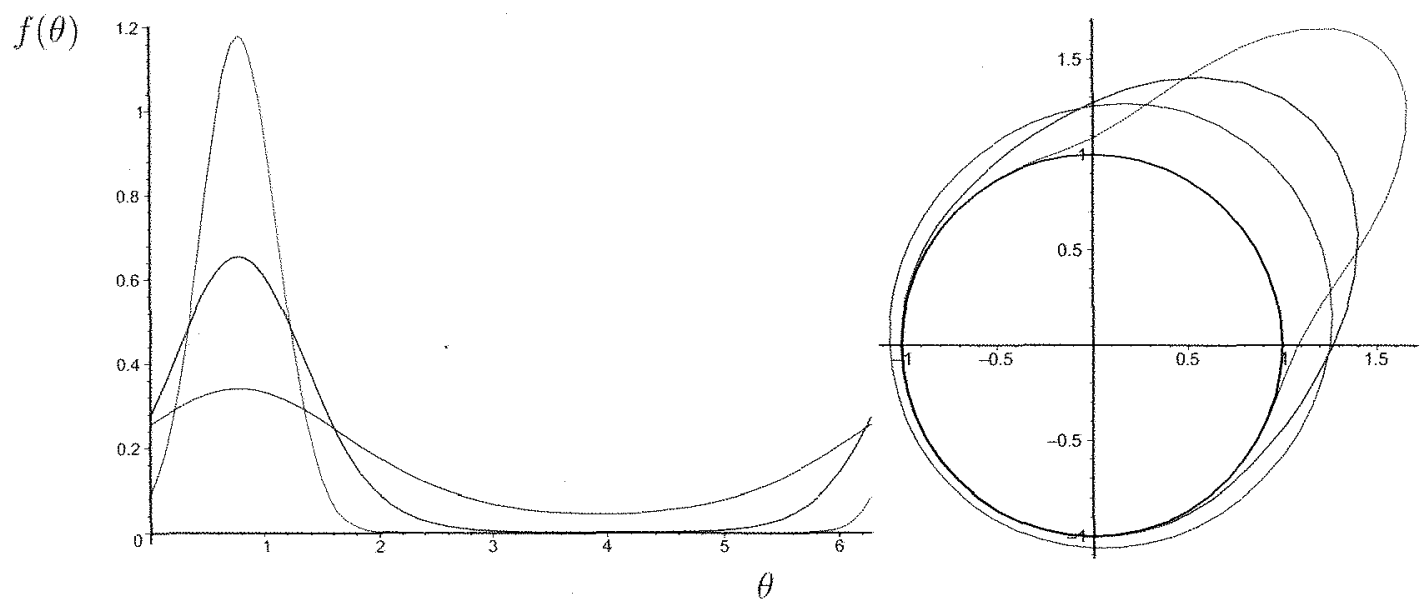

Figura 7 - Funções densidade de probabilidade von Mises com $\mu=\frac{\pi}{4}$ e parâmetros de concentração $k=1$ (vermelho), $k=3$ (azul) e $k=9$ (verde), na reta real (à esquerda) e na circuferência (à direita)

cujo logaritmo é

$$
l(\mu, \kappa)=-n \log 2 \pi-n \log I_{0}(\kappa)+\kappa \sum_{i=1}^{n} \cos \left(\theta_{i}-\mu\right) .
$$

Os estimadores de máxima verossimilhança $\hat{\mu}$ de $\mu$ e $\hat{\kappa}$ de $\kappa$ são definidos, então, como os valores de $\mu$ e $\kappa$ que maximizam a função (10) ou, equivalentemente, a função (11). Para obtê-los, deve-se solucionar o sistema de equações formado pelas equações $\frac{\partial l(\mu, \kappa)}{\partial \mu}=0 \mathrm{e} \frac{\partial l(\mu, \kappa)}{\partial \kappa}=0$, ou seja, encontrar $\hat{\mu}$ e $\hat{\kappa}$ tais que

$$
\hat{\kappa} \sum_{i=1}^{n} \operatorname{sen}\left(\theta_{i}-\hat{\mu}\right)=0
$$

$\mathrm{e}$

$$
\begin{gathered}
-n \frac{I_{0}^{\prime}(\hat{\kappa})}{I_{0}(\hat{\kappa})}+\sum_{i=1}^{n} \cos \left(\theta_{i}-\hat{\mu}\right)=0 . \\
\text { Uma vez que } \\
I_{0}^{\prime}(\kappa)=\frac{\partial I_{0}(\kappa)}{\partial \kappa}=\sum_{r=1}^{\infty} \frac{1}{r !^{2}}(2 r)\left(\frac{1}{2} \kappa\right)^{2 r-1} \frac{1}{2}=\sum_{r=1}^{\infty} \frac{1}{(r-1) ! r !}\left(\frac{1}{2} \kappa\right)^{2 r-1}
\end{gathered}
$$


fazendo-se $s=r-1$, tem-se

$$
I_{0}^{\prime}(\kappa)=\sum_{s=0}^{\infty} \frac{1}{s !(s+1) !}\left(\frac{1}{2} \kappa\right)^{2 s+1}=I_{1}(\kappa) .
$$

Desse modo, a eq. (13) pode ser reescrita da seguinte forma

$$
-n \frac{I_{1}(\hat{\kappa})}{I_{0}(\hat{\kappa})}+\sum_{i=1}^{n} \cos \left(\theta_{i}-\hat{\mu}\right)=0
$$

ou seja,

$$
-n A_{1}(\hat{\kappa})+\sum_{i=1}^{n} \cos \left(\theta_{i}-\hat{\mu}\right)=0 .
$$

A partir das equações (12) e (2), obtém-se

$$
\begin{aligned}
\hat{\kappa} \sum_{i=1}^{n} \operatorname{sen}\left(\theta_{i}-\hat{\mu}\right) & =0 \\
\sum_{i=1}^{n} \operatorname{sen}\left(\theta_{i}-\hat{\mu}\right) & =0 \\
\sum_{i=1}^{n} \operatorname{sen} \theta_{i} \cos \hat{\mu}-\sum_{i=1}^{n} \cos \theta_{i} \operatorname{sen} \hat{\mu} & =0 \\
\sum_{i=1}^{n} \operatorname{sen} \theta_{i} \cos \hat{\mu} & =\sum_{i=1}^{n} \cos \theta_{i} \operatorname{sen} \hat{\mu} \\
\frac{\operatorname{sen} \hat{\mu}}{\cos \hat{\mu}} & =\frac{\sum_{i=1}^{n} \operatorname{sen} \theta_{i}}{\sum_{i=1}^{n} \cos \theta_{i}} \overline{\bar{S}} \\
\operatorname{tg} \hat{\mu} & =\operatorname{tg} \bar{x}_{0} \\
\hat{\mu} & =\bar{x}_{0} .
\end{aligned}
$$

Conclui-se, desta forma, que $\hat{\mu}=\bar{x}_{0}$ é a solução da eq. (12) e, conforme será demonstrado adiante, maximiza as funções (10) e (11). Assim, o ângulo médio $\bar{x}_{0}$, dado pela eq. (3), é o estimador de máxima verossimilhança do parâmetro $\mu$.

Segundo Bingham \& Mardia ${ }^{3}$, citado por Batschelet (1981), a distribuição de von Mises é a única distribuição circular cujo estimador de máxima

\footnotetext{
${ }^{3}$ BINGHAM, M.S., MARDIA, K.V. Maximum likelihood characterization of the von Mises
} distribuition. In PATIL, C.P.; Reidel; Dordrecht (Ed.). Statistical Distribuitions in Scientific Work, v.3 p.387-398, 1975 
verossimilhança do ângulo médio populacional é igual ao ângulo médio amostral.

Partindo-se da eq. (15), tem-se que

$$
\begin{aligned}
A_{1}(\hat{\kappa}) & =\frac{1}{n} \sum_{i=1}^{n} \cos \left(\theta_{i}-\hat{\mu}\right) \\
& =\frac{1}{n} \sum_{i=1}^{n}\left[\cos \theta_{i} \cos \hat{\mu}+\operatorname{sen} \theta_{i} \operatorname{sen} \hat{\mu}\right] \\
& =\frac{1}{n}\left(\cos \hat{\mu} \sum_{i=1}^{n} \cos \theta_{i}+\operatorname{sen} \hat{\mu} \sum_{i=1}^{n} \operatorname{sen} \theta i\right) \\
& =\cos \hat{\mu} \bar{C}+\operatorname{sen} \hat{\mu} \bar{S}
\end{aligned}
$$

Mas,

$$
\operatorname{sen} \hat{\mu}=\operatorname{sen} \bar{x}_{0}=\frac{\bar{S}}{\bar{R}}
$$

e

$$
\cos \hat{\mu}=\cos \bar{x}_{0}=\frac{\bar{C}}{\bar{R}}
$$

Logo, substituindo-se (18) e (19) na eq. (17), obtêm-se

$$
A_{1}(\hat{\kappa})=\frac{\bar{C}}{\bar{R}} \bar{C}+\frac{\bar{S}}{\bar{R}} \bar{S}=\frac{1}{\bar{R}}\left[\bar{C}^{2}+\bar{S}^{2}\right]=\frac{\bar{R}^{2}}{\bar{R}}=\bar{R}
$$

e

$$
\hat{\kappa}=A_{1}^{-1}(\bar{R})
$$

sendo $A_{1}^{-1}$ a função inversa da função $A_{1}$. Conclui-se, portanto, que $\hat{\kappa}=A_{1}^{-1}(\bar{R})$ é solução da eq. (13) e, como será visto adiante, maximiza as funções (10) e (11), sendo assim, o estimador de máxima verossimilhança para o parâmetro de concentração $\kappa$. Como ilustração, a Figura 8 apresenta o gráfico da função (20) para $0<\bar{R} \leq 0,9$.

Convém observar que a solução (20) da eq. (13) só pode ser obtida numericamente. Assim, Mardia (1972) apresenta uma tabela com alguns valores selecionados de $A_{1}$ e $A_{1}^{-1}$. Para valores não apresentados nessa tabela, sugere a utilização de aproximações propostas por autores como Abramowitz e Stegun (1965), que se baseiam em expansōes da função $A_{1}(\kappa)$ em séries e posterior inversão das mesmas. 


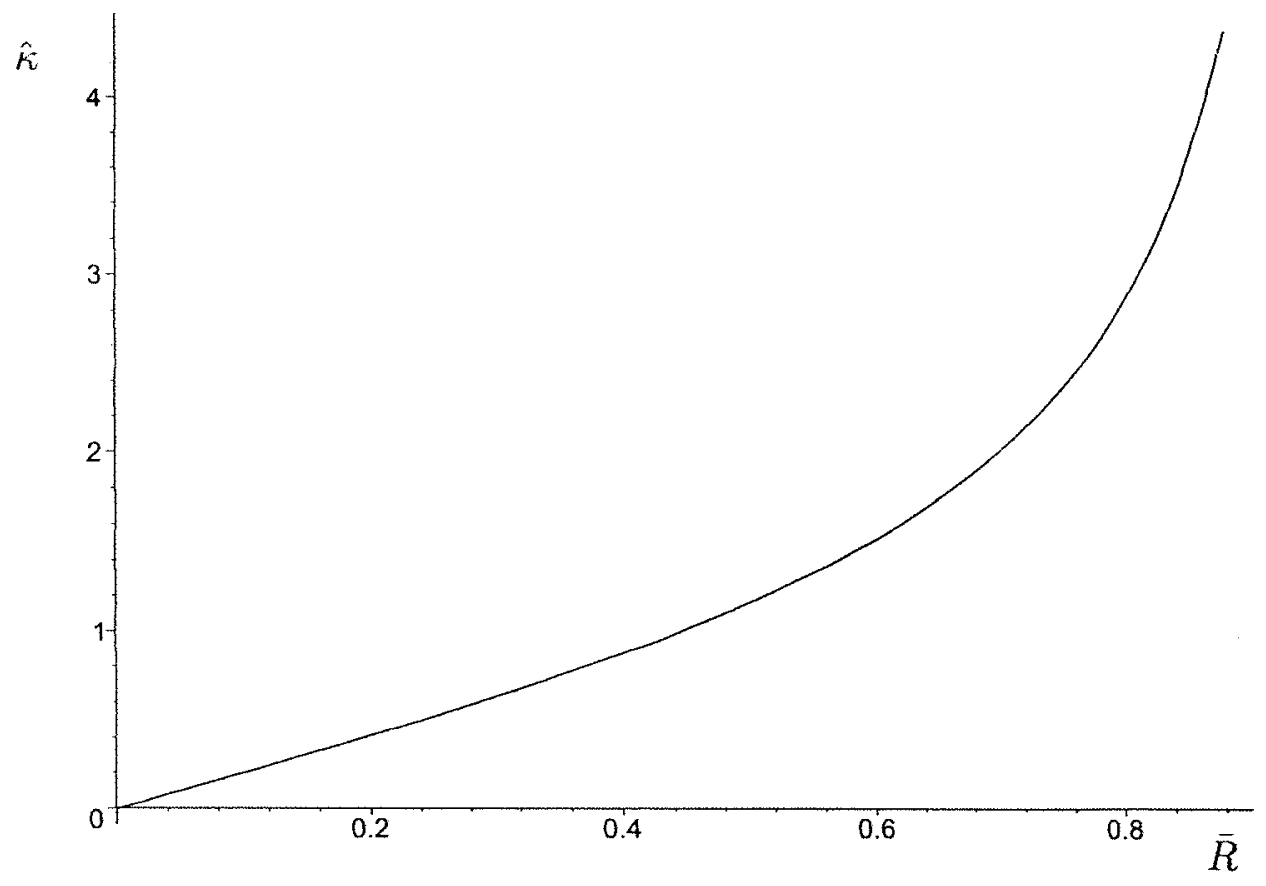

Figura 8 - Gráfico da função $\hat{\kappa}=A_{1}^{-1}(\bar{R})$, para $0<\bar{R} \leq 0,9$

Uma aproximação simples para o estimador $\hat{\kappa}$, apresentada por Best \& Fisher (1981), é dada por

$$
A^{-1}(\bar{R}) \approx\left\{\begin{array}{ll}
2 \bar{R}+\bar{R}^{3}+\frac{5 \bar{R}^{5}}{6}, & \text { se } \bar{R}<0,53 \\
-0,4+1,39 \bar{R}+\frac{0,43}{(1-\bar{R})}, & \text { se } 0,53 \leq \bar{R}<0,85 . \\
\left(\bar{R}^{3}-4 \bar{R}^{2}+3 \bar{R}\right)^{-1}, & \text { se } \bar{R} \geq 0,85
\end{array} .\right.
$$

No entanto, Best \& Fisher (1981) concluem que para amostras pequenas $(n \leq 15)$ e valores pequenos de $\bar{R}(\bar{R}<0,7)$ o estimador de $\kappa$ é viesado, superestimando o verdadeiro valor de $\kappa$ e sugerem, para este caso, a utilização do seguinte estimador corrigido

$$
\hat{\kappa}^{*}=\left\{\begin{array}{ll}
\operatorname{máx}\left(\hat{\kappa}-2(n \hat{\kappa})^{-1}, 0\right) & \text { se } \hat{\kappa}<2 \\
\frac{(n-1)^{3} \hat{\kappa}}{\left(n^{3}+n\right)}, & \text { se } \hat{\kappa} \geq 2
\end{array} .\right.
$$

A seguir, será demonstrado que as soluções (16) e (20), obtidas para as equações (12) e (13), maximizam a função (11), ou seja, são estimadores de máxima 
verossimilhança de $\mu$ e $\kappa$. Para isso, deve-se verificar se o determinante da matriz de derivadas segundas $H$, dada por

$$
H=\left[\begin{array}{ll}
\frac{\partial^{2} l(\mu, \kappa)}{\partial \mu^{2}} & \frac{\partial^{2} l(\mu, \kappa)}{\partial \mu \partial \kappa} \\
\frac{\partial^{2} l(\mu, \kappa)}{\partial \kappa \partial \mu} & \frac{\partial^{2} l(\mu, \kappa)}{\partial \kappa^{2}}
\end{array}\right]
$$

é positivo e $H_{11}$ é negativo, para $\mu=\hat{\mu}$ e $\kappa=\hat{\kappa}$. Os elementos da matriz $H$ assim definida, são

$$
\begin{aligned}
& H_{11}=-\sum_{i=1}^{n} \kappa \cos \left(\theta_{i}-\mu\right)=-\kappa \sum_{i=1}^{n}\left(\cos \theta_{i} \cos \mu+\operatorname{sen} \theta_{i} \operatorname{sen} \mu\right) \\
&=-\kappa\left(\cos \mu \sum_{i=1}^{n} \cos \theta_{i}+\operatorname{sen} \mu \sum_{i=1}^{n} \operatorname{sen} \theta_{i}\right) \\
&=-n \kappa(\cos \mu \bar{C}+\operatorname{sen} \mu \bar{S}), \\
& H_{12}=H_{21}=\sum_{i=1}^{n} \operatorname{sen}\left(\theta_{i}-\mu\right)=\sum_{i=1}^{n}\left(\operatorname{sen} \theta_{i} \cos \mu-\cos \theta_{i} \operatorname{sen} \mu\right) \\
&=\cos \mu \sum_{i=1}^{n} \operatorname{sen} \theta_{i}-\operatorname{sen} \mu \sum_{i=1}^{n} \cos \theta_{i} \\
&=\cos \mu \bar{S}-\operatorname{sen} \mu \bar{C}
\end{aligned}
$$

e

$$
H_{22}=-n A_{1}^{\prime}(\kappa)=-n\left[-A_{1}^{2}(\kappa)-\frac{1}{\kappa} A_{1}(\kappa)+1\right] .
$$

Logo, tem-se que

$$
\begin{aligned}
\left.H_{11}\right|_{\mu=\hat{\mu}, \kappa=\hat{\kappa}} & =-n \hat{\kappa}\left(\frac{\bar{C}}{\bar{R}} \bar{C}+\frac{\bar{S}}{\bar{R}} \bar{S}\right)=-n \hat{\kappa} \frac{\bar{R}^{2}}{\bar{R}}=-n \hat{\kappa} \bar{R}, \\
\left.H_{12}\right|_{\mu=\hat{\mu}, \kappa=\hat{\kappa}}=\left.H_{21}\right|_{\mu=\hat{\mu}, \kappa=\hat{\kappa}} & =\frac{\bar{C}}{\bar{R}} \bar{S}-\frac{\bar{S}}{\bar{R}} \bar{C}=0 \\
\left.H_{22}\right|_{\mu=\hat{\mu}, \kappa=\hat{\kappa}} & =-n A_{1}^{\prime}(\hat{\kappa})=-n\left[-\bar{R}^{2}-\frac{1}{\hat{\kappa}} \bar{R}+1\right]
\end{aligned}
$$

e portanto,

$$
\left.H\right|_{\mu=\hat{\mu}, \kappa=\hat{\kappa}}=\left[\begin{array}{cc}
-n \hat{\kappa} \bar{R} & 0 \\
0 & -n A_{1}^{\prime}(\hat{\kappa})
\end{array}\right]
$$


cujo determinante é

$$
n^{2} \hat{\kappa} \bar{R} A_{1}^{\prime}(\hat{\kappa})
$$

A função $A_{1}(\kappa)$, por sua vez, é sempre crescente e, portanto, sua derivada $A_{1}^{\prime}(\kappa)$ é sempre positiva. Logo, como $\hat{\kappa}>0$ e $0<\bar{R} \leq 1$, o determinante de $H$ é maior do que zero e $H_{11}$ é menor do que zero para $\mu=\hat{\mu}$ e $\kappa=\hat{\kappa}$. Portanto, as soluções (16) e (20) maximizam a função (11), ou seja, são estimadores de máxima verossimilhança de $\mu$ e $\kappa$.

Sejam,

$$
\theta_{j 1}, \ldots, \theta_{j n_{j}}, ; j=1, \ldots, q
$$

$q$ amostras independentes de tamanhos $n_{j}$ de distribuiçōes de von Mises com parâmetros $\mu_{j}$ e $\kappa_{j}$ obtidas, por exemplo, a partir de um ensaio inteiramente ao acaso com $q$ tratamentos e $n_{j}$ repetições por tratamento, conforme ilustra a Tabela 3.

Tabela 3. Resultados de um ensaio inteiramente ao acaso com $q$ tratamentos e $n_{j}$ $(j=1, \ldots, q)$ repetições por tratamento

\begin{tabular}{cccc}
\hline \multicolumn{4}{c}{ Tratamentos } \\
1 & 2 & $\cdots$ & $q$ \\
\hline$\theta_{11}$ & $\theta_{21}$ & $\cdots$ & $\theta_{q 1}$ \\
$\theta_{12}$ & $\theta_{22}$ & $\cdots$ & $\theta_{q 2}$ \\
$\theta_{13}$ & $\theta_{23}$ & $\cdots$ & $\theta_{q 3}$ \\
$\cdots$ & $\cdots$ & $\cdots$ & $\cdots$ \\
$\theta_{1 n_{1}}$ & $\theta_{2 n_{2}}$ & $\cdots$ & $\theta_{q n_{q}}$ \\
\hline
\end{tabular}

Considere que, para o $j$-ésimo tratamento,

$$
\begin{aligned}
& S_{j}=\sum_{i=1}^{n_{j}} \operatorname{sen} \theta_{j i}, \\
& C_{j}=\sum_{i=1}^{n_{j}} \cos \theta_{j i},
\end{aligned}
$$




$$
\begin{gathered}
R_{j}=\sqrt{C_{j}^{2}+S_{j}^{2}}, \\
\bar{R}_{j}=\frac{R_{j}}{n_{j}} \\
\hat{\kappa}_{j}=A_{1}^{-1}\left(\bar{R}_{j}\right)
\end{gathered}
$$

e

$$
\hat{\mu}_{j}=\bar{x}_{0 j}
$$

obtido por meio da função (3).

$$
\begin{aligned}
& \text { Considerando, então, o conjunto total de } n=\sum_{j=1}^{q} n_{j} \text { dados, sejam } \\
& S=\sum_{j=1}^{q} S_{j} \\
& C=\sum_{j=1}^{q} C_{j} \\
& R=\sqrt{C^{2}+S^{2}} \text {, } \\
& \bar{R}=\frac{R}{n}, \\
& \bar{R}^{\prime}=\frac{1}{n} \sum_{j=1}^{q} R_{j} \\
& \hat{\kappa}=A_{1}^{-1}(\bar{R}), \\
& \tilde{\kappa}=A_{1}^{-1}\left(\bar{R}^{\prime}\right), \\
& \hat{\mu}=\bar{x}_{0},
\end{aligned}
$$

obtido por meio da função (3).

Suponha que se queira testar a hipótese

$$
H_{0}: \mu_{1}=\mu_{2}=\cdots=\mu_{q}
$$

contra a hipótese de que ao menos um dos parâmetros de locação seja diferente de outro, supondo que $\kappa_{1}=\kappa_{2}=\cdots=\kappa_{q}=\kappa, \kappa$ desconhecido. 
Segundo Stephens (1972), para $q \geq 2$ e $\tilde{\kappa} \geq 2$ (ou $\bar{R}^{\prime} \geq 0,45$ ), a estatística

$$
F=\left(1+\frac{3}{8 \tilde{\kappa}}\right) \frac{n-q}{q-1} \frac{\sum_{j=1}^{q} R_{j}-R}{n-\sum_{j=1}^{q} R_{j}}
$$

tem, sob $H_{0}$, distribuição aproximadamente $F_{q-1, n-q}$. Logo, dado um certo nível de significância $\alpha$ e o valor da estatística para a amostra, rejeita-se $H_{0}$ se

$$
\operatorname{Pr}\left(F_{q-1, n-q}>F\right) \leq \alpha .
$$

Nesse caso, a análise pode ser apresentada na forma de uma tabela usual de análise de variância, como ilustra a Tabela 4 , sendo $C V$ a causa de variação, $G L$ o número de graus de liberdade, $S Q$ a soma de quadrados e $Q M$ o quadrado médio.

Tabela 4. Análise de variância para o teste da hipótese $H_{0}: \mu_{1}=\cdots=\mu_{q}$, supondo $\tilde{\kappa} \geq 2\left(\right.$ ou $\left.\bar{R}^{\prime} \geq 0,45\right)$

\begin{tabular}{lcccc}
\hline CV & $G L$ & $S Q$ & $Q M$ & $F$ \\
\hline Tratamentos & $q-1$ & $\sum_{j=1}^{q} R_{j}-R$ & $\frac{\sum_{j=1}^{q} R_{j}-R}{q-1}$ & $\left(1+\frac{3}{8 \tilde{\kappa}}\right) \frac{n-q}{q-1} \frac{\sum_{j=1}^{q} R_{j}-R}{n-\sum_{j=1}^{q} R_{j}}$ \\
& & & \\
Resíduo & $n-q$ & $n-\sum_{j=1}^{q} R_{j}$ & $\frac{n-\sum_{j=1}^{q} R_{j}}{n-q}$ & - \\
\hline Total & $n-1$ & $n-R$ & - & - \\
\hline
\end{tabular}

Este teste foi originalmente proposto por Watson \& Williams (1956) sem o fator de correção $\left(1+\frac{3}{8 \tilde{\kappa}}\right)$, apresentado posteriormente por Stephens (1972) de modo a reduzir o erro de aproximação.

Para $\tilde{\kappa}<2$ (ou $\bar{R}^{\prime}<0,45$ ), no entanto, Mardia (1972) sugere que se 
faça o teste utilizando-se a estatística

$$
Q=\frac{2}{n}\left[\frac{\left(\sum_{j=1}^{q} R_{j}\right)^{2}-R^{2}}{1-\frac{1}{8} \kappa^{2}+\frac{q}{2 n \kappa^{2}}}\right]
$$

que tem sob $H_{0}$, distribuição aproximadamente $\chi_{q-1}^{2}$. Sugere, na prática, que se substitua $\kappa$ por $\tilde{\kappa}$ e assim, dados o valor da estatística para uma certa amostra e o nível de significância $\alpha$, rejeita-se $H_{0}$ se $\operatorname{Pr}\left(\chi_{q-1}^{2}>Q\right) \leq \alpha$.

McGuirre (1992) sugere, por outro lado, que, para dados de tonalidade, se faça a análise usual de variância.

A análise de variância circular de um ensaio inteiramente ao acaso com $q$ tratamentos, como visto anteriormente, é usada para testar a hipótese (23). Contudo, a rejeição da hipótese $H_{0}$ não implica em que todas as $q$ médias dos tratamentos sejam diferentes entre si. Assim, pode-se estar interessado em um procedimento de comparação múltiplas das médias dos $q$ tratamentos. Deste modo, suponha, que se queira testar a hipótese da igualdade das médias $\mu_{j}$ e $\mu_{j^{\prime}}$, referentes ao tratamentos $j$ e $j^{\prime}, \operatorname{com} j<j^{\prime}$, ou seja, testar a hipótese

$$
H_{0}: \mu_{j}=\mu_{j^{\prime}}
$$

contra a hipótese alternativa

$$
H_{a}: \mu_{j} \neq \mu_{j^{\prime}}
$$

Sejam

$$
\begin{aligned}
S_{j j^{\prime}}=S_{j}+S_{j^{\prime}} & =\sum_{i=1}^{n_{j}} \operatorname{sen} \theta_{j i}+\sum_{i=1}^{n_{j^{\prime}}} \operatorname{sen} \theta_{j^{\prime} i} \\
C_{j j^{\prime}}=C_{j}+C_{j^{\prime}} & =\sum_{i=1}^{n_{j}} \cos \theta_{j i}+\sum_{i=1}^{n_{j^{\prime}}} \cos \theta_{j^{\prime} i} \\
R_{j j^{\prime}} & =\sqrt{C_{j j^{\prime}}^{2}+S_{j j^{\prime}}^{2}}
\end{aligned}
$$

Para $\tilde{\kappa} \geq 2$ (ou $\bar{R}^{\prime} \geq 0,45$ ), a estatística

$$
F_{j j^{\prime}}=\left(1+\frac{3}{8 \tilde{\kappa}}\right) \frac{(n-q)\left(R_{j}+R_{j^{\prime}}-R_{j j^{\prime}}\right)}{\left(n-\sum_{j=1}^{q} R_{j}\right)}
$$


tem, sob $H_{0}$, distribuição aproximadamente $F_{1 ; n-q}$. Logo, dado um certo nível de significância $\alpha$ e o valor da estatística $F_{j j^{\prime}}$, rejeita-se $H_{0}$ se $\operatorname{Pr}\left(F_{1 ; n-q}>F_{j j^{\prime}}\right) \leq \alpha$.

Para o caso em que $\tilde{\kappa}<2$ (ou $\bar{R}^{\prime}<0,45$ ), sugere-se que se use a estatística

$$
Q_{j j^{\prime}}=\frac{n}{2}\left[\frac{\left(R_{j}+R_{j^{\prime}}\right)^{2}-R_{j j^{\prime}}^{2}}{1+\frac{1}{8} \kappa^{2}+\frac{q}{2 n \kappa^{2}}}\right]
$$

que tem, sob $H_{0}$, distribuição aproximadamente $\chi_{1}^{2}$. Sugere-se que se substitua $\kappa$ por $\tilde{\kappa}$ e assim, dados o valor da estatística e o nível de significância $\alpha$, rejeita-se $H_{0}$ se $\operatorname{Pr}\left(\chi_{1}^{2}>Q_{j j^{\prime}}\right) \leq \alpha$.

McGuire (1992), no entanto, sugere que se utilizem os métodos usuais de comparações múltiplas para a comparação das médias, duas a duas, cita por exemplo, o teste de Ryan-Einot-Gabriel-Welsh.

Considere a diferença absoluta entre os ângulos médios $\hat{\mu}_{j}$ e $\hat{\mu}_{j^{\prime}}$, como

$$
d_{j j^{\prime}}=\min \left(\left|\hat{\mu}_{j}-\hat{\mu}_{j^{\prime}}\right|, 2 \pi-\left|\hat{\mu}_{j}-\hat{\mu}_{j^{\prime}}\right|\right)
$$

Os resultados podem ser apresentados, então, em uma tabela como ilustra a Tabela 5 sendo que a probabilidade de se cometer o erro do tipo I, ou valor-p é calculado por

$$
\text { valor- } \mathrm{p}_{j j^{\prime}}=\operatorname{Pr}\left(F_{1 ; n-q} \geq F_{j j^{\prime}}\right)
$$

ou

$$
\text { valor- } \mathrm{p}_{j j^{\prime}}=\operatorname{Pr}\left(\chi_{1}^{2} \geq Q_{j j^{\prime}}\right)
$$

, para os casos de utilização das estatísticas (25) ou (26), respectivamente.

Convém ressaltar que para o procedimento de comparações múltiplas apresentado aqui, são realizados, no total, $\frac{q(q-1)}{2}$ testes de hipóteses. Assim, a probabilidade de se cometer pelo menos uma vez o erro do tipo I (rejeitar a hipótese $H_{0}: \mu_{j}=\mu_{j^{\prime}}$, quando $H_{0}$ é verdadeira) nestes $\frac{q(q-1)}{2}$ testes, será sempre maior do que $\alpha$. Considere que o nível de significância adotado para cada um dos testes seja $\alpha$, ou seja, que a probabilidade máxima aceitável de se cometer o erro do tipo I, para 
Tabela 5. Resultados do teste de comparações múltiplas para os dados da Tabela 3

\begin{tabular}{|c|c|c|c|c|c|}
\hline \multirow{2}{*}{$\begin{array}{c}\text { Tratamentos } \\
j, j^{\prime}\end{array}$} & \multicolumn{2}{|c|}{$\begin{array}{l}\text { Ângulos } \\
\text { médios }\end{array}$} & \multirow{2}{*}{$\begin{array}{c}\text { Diferença } \\
\text { absoluta } \\
d_{j j^{\prime}} \\
\end{array}$} & \multirow{2}{*}{$\begin{array}{l}\text { Estatística } \\
F_{j j^{\prime}} \text { ou } Q_{j j^{\prime}}\end{array}$} & \multirow[t]{2}{*}{ valor- $\mathrm{p}_{j j^{\prime}}$} \\
\hline & $\hat{\mu}_{j}$ & $\hat{\mu}_{j^{\prime}}$ & & & \\
\hline 1,2 & $\hat{\mu}_{1}$ & $\hat{\mu}_{2}$ & $d_{12}$ & $F_{12}$ ou $Q_{12}$ & valor-p $\mathrm{p}_{12}$ \\
\hline 1,3 & $\hat{\mu}_{1}$ & $\hat{\mu}_{3}$ & $d_{13}$ & $F_{13}$ ou $Q_{13}$ & valor- $\mathrm{p}_{13}$ \\
\hline$\cdots$ & . & & $\cdots$ & $\cdots$ & $\cdots$ \\
\hline $1, q$ & $\hat{\mu}_{1}$ & $\hat{\mu}_{q}$ & $d_{1 q}$ & $F_{1 q}$ ou $Q_{1 q}$ & valor $-\mathrm{p}_{1 q}$ \\
\hline 2,3 & $\hat{\mu}_{2}$ & $\hat{\mu}_{3}$ & $d_{23}$ & $F_{23}$ ou $Q_{23}$ & valor- $\mathrm{p}_{23}$ \\
\hline 2,4 & $\hat{\mu}_{2}$ & $\hat{\mu}_{4}$ & $d_{24}$ & $F_{24}$ ou $Q_{24}$ & valor- $\mathrm{p}_{24}$ \\
\hline$\cdots$ & . & & $\cdots$ & $\cdots$ & $\cdots$ \\
\hline $2, q$ & $\hat{\mu}_{2}$ & $\hat{\mu}_{q}$ & $d_{2 q}$ & $F_{2 q}$ ou $Q_{2 q}$ & valor- $\mathrm{p}_{2 q}$ \\
\hline$\cdots$ & $\cdots$ & & $\ldots$ & $\ldots$ & $\cdots$ \\
\hline$q-1, q$ & $\hat{\mu}_{q-1}$ & $\hat{\mu}_{q}$ & $d_{q-1, q}$ & $F_{q-1, q}$ ou $Q_{q-1, q}$ & valor- $\mathrm{p}_{q-1, q}$ \\
\hline
\end{tabular}

cada um dos testes, seja $\alpha$. Supondo que, pelo menos aproximadamente, os resultados dos testes sejam independentes, então a probabilidade $\alpha^{*}$ máxima aceitável de se cometer o erro do tipo I em pelo menos um dos testes será dada por:

$$
\alpha^{*}=1-(1-\alpha)^{\frac{q(q-1)}{2}} .
$$

Caso se deseje fixar $\alpha^{*}$, então, para cada teste da hipótese $H_{0}: \mu_{j}=\mu_{j^{\prime}}$, deve-se adotar o nível de significância

$$
\alpha=1-\left(1-\alpha^{*}\right)^{\frac{2}{q(q-1)}}
$$

Assim, por exemplo, para $q=4$ tratamentos e $\alpha^{*}=0,05$ deverá ser adotado o nível de significância $\alpha=1-0,95^{\frac{1}{6}}=0,0085$ em cada teste da hipótese $H_{0}: \mu_{j}=\mu_{j^{\prime}}$. Uma alternativa a este caso, seria considerar, para cada teste o novo 
valor-p dado por

$$
\text { valor }-\mathrm{p}^{*}=1-(1-\text { valor }-\mathrm{p}) \frac{q(q-1)}{2}
$$

e rejeitar a hipótese de nulidade se valor- $\mathrm{p}^{*} \leq \alpha^{*}$. Tais procedimentos, bastante conservadores, merecem, no entanto, estudos mais aprofundados.

Convém ressaltar que para a realização dos testes apresentados até aqui, pressupõe-se que as amostras sejam independentes, extraídas de populações de von Mises com parâmetros de concentração iguais.

De modo a se testar a pressuposição de igualdades dos parâmetros de concentração, será apresentado, a seguir o procedimento proposto por Mardia (1972) para o teste da hipótese

$$
H_{0}: \kappa_{1}=\ldots=\kappa_{q}
$$

contra a hipótese de que ao menos um dos parâmetros de concentração difere de outro.

Este procedimento é dividido em três casos:

Caso $1 \bar{R}^{\prime}<0,45$. Considere a estatística $U_{1}$ dada por

$$
U_{1}=\sum_{j=1}^{q} w_{j} g_{1}^{2}\left(\tilde{R}_{j}\right)-\frac{\left[\sum_{j=1}^{q} w_{j} g_{1}\left(\tilde{R}_{j}\right)\right]^{2}}{\sum_{j=1}^{q} w_{j}}
$$

em que

$$
\begin{gathered}
\tilde{R}_{j}=2 \bar{R}_{j} \\
g_{1}\left(\tilde{R}_{j}\right)=\operatorname{arcsen}\left[\left(\frac{3}{8}\right)^{\frac{1}{2}} \tilde{R}_{j}\right]
\end{gathered}
$$

e

$$
w_{j}=\frac{4}{3}\left(n_{j}-4\right)
$$

Segundo Mardia (1972), $U_{1}$ tem, sob $H_{0}$, distribuição aproximada $\chi_{q-1}^{2}$. Logo, dados um nível de significância $\alpha$ e o valor da estatística para a amostra, rejeita-se $H_{0}$ se $\operatorname{Pr}\left(\chi_{q-1}^{2}>U_{1}\right) \leq \alpha$. 
Caso $20,45 \leq \bar{R}^{\prime}<0,70$. Considere a estatística $U_{2}$ dada por

$$
U_{2}=\sum_{j=1}^{q} w_{j} g_{2}^{2}\left(\bar{R}_{j}\right)-\frac{\left[\sum_{j=1}^{q} w_{j} g_{2}\left(\bar{R}_{j}\right)\right]^{2}}{\sum_{j=1}^{q} w_{j}},
$$

em que

$$
g_{2}\left(\vec{R}_{j}\right)=\operatorname{arcsenh} \frac{\bar{R}_{j}-1,08940}{0,25789}
$$

$\mathrm{e}$

$$
w_{j}=\frac{n_{j}-3}{0,7979} .
$$

Segundo Mardia (1972), $U_{2}$ tem, sob $H_{0}$, distribuição aproximada $\chi_{q-1}^{2}$. Logo, dados um nível de significância $\alpha$ e o valor da estatística para a amostra, rejeita-se $H_{0}$ se $\operatorname{Pr}\left(\chi_{q-1}^{2}>U_{2}\right) \leq \alpha$.

Caso $3 \bar{R}^{\prime} \geq 0,70$. Neste caso, sob a hipótese $H_{0}$, têm-se que

$$
2 \kappa\left(n_{j}-R_{j}\right) \sim \chi_{n_{j}-1}^{2}
$$

o que implica que esta quantidade tem um comportamento semelhante à variância amostral da teoria normal, podendo-se usar o teste de Bartlett. Assim, considere a estatística $U_{3}$ dada por

$$
U_{3}=\frac{1}{1+d}\left[\nu \log \frac{n-\sum_{j=1}^{q} R_{j}}{\nu}-\sum_{j=1}^{q} \nu_{j} \log \frac{n_{j}-R_{j}}{\nu_{j}}\right]
$$

em que

$$
\begin{gathered}
\nu_{j}=n_{j}-1, \\
\nu=n-q
\end{gathered}
$$

$\mathrm{e}$

$$
d=\frac{\sum_{j=1}^{q} \frac{1}{\nu_{j}}-\frac{1}{\nu}}{3(q-1)} .
$$


Segundo Mardia (1972), $U_{3}$ tem, sob $H_{0}$, distribuição aproximada $\chi_{q-1}^{2}$. Logo, dados um nível de significância $\alpha$ e o valor da estatística para a amostra, rejeita-se $H_{0}$ se $\operatorname{Pr}\left(\chi_{q-1}^{2}>U_{3}\right) \leq \alpha$.

Além da pressuposição de homogeneidade dos parâmetros de concentração, para o teste da hipótese (23), é necessário verificar se a distribuição de von Mises ajusta-se ao conjunto de dados, o que será visto a seguir.

Considere uma amostra aleatória $\theta_{1}, \ldots, \theta_{n}$ de uma variável $\Theta$ com distribuição angular desconhecida. Sejam:

(i) $\theta_{i}^{*}=\left(\theta_{i}-\hat{\mu}+\pi\right)[\bmod 2 \pi], \quad i=1, \ldots, n$;

(ii) $\theta_{[i]}^{*}$ o i-ésimo elemento de $\left\{\theta_{1}, \ldots, \theta_{n}\right\}$ colocados em ordem crescente;

(iii) $F_{\Theta}^{-1}(P)$ a função inversa da função de distribuição de von Mises (9) com $\mu=\pi$, $\kappa=\hat{\kappa}$ e $P$ uma constante entre 0 e 1 .

Propõe-se, então, que se construa o gráfico quantil-quantil, definido como o gráfico formado pelos pontos de coordenadas

$$
\left[\theta_{[i]}^{*}+\hat{\mu}-\pi ; F_{\Theta}^{-1}\left(\frac{i}{n+1}\right)+\hat{\mu}-\pi\right], \quad i=1, \ldots, n .
$$

Para este caso, quanto mais próximos da reta passando pela origem e com coeficiente angular um, estiverem os pontos, maior a evidência de que $\Theta$ tenha distribuição de von Mises com parâmetros $\mu$ e $\kappa$.

Considere, por outro lado, os resultados $\theta_{j i}$ de um experimento inteiramente ao acaso, apresentados na Tabela 3. O procedimento anterior pode ser utilizado como uma forma empírica de se verificar a pressuposição de que as $q$ amostras foram extraídas de populações com distribuições de von Mises com parâmetros de concentração iguais. Propõe-se nesse caso, construir um gráfico quantil-quantil, seguindo o procedimento anterior, para o conjunto de valores

$$
\left\{\epsilon_{11}, \epsilon_{12}, \ldots, \epsilon_{1 n_{1}}, \epsilon_{21}, \epsilon_{22}, \ldots, \epsilon_{2 n_{2}}, \ldots, \epsilon_{q 1}, \epsilon_{q 2}, \ldots, \epsilon_{q n_{q}}\right\}
$$


sendo

$$
\begin{aligned}
\epsilon_{j i}=\theta_{j i}-\hat{\mu}_{j}, \quad j & =1, \ldots, q i=1, \ldots, n_{j}, \\
n & =\sum_{j=1}^{q} n_{j}
\end{aligned}
$$

e

$$
\kappa=\tilde{\kappa}
$$

De forma análoga ao caso anterior, quanto mais próximos da reta passando pela origem e com coeficiente angular um, estiverem os pontos do gráfico quantil-quantil, maior a evidência de que os dados provenham de distribuições de von Mises com parâmetros de concentração iguais.

Um teste estatístico apropriado para amostras aleatórias de tamanho superior a 20 foi proposto por Lockhart \& Stephens (1985) e será descrito a seguir.

Seja $\left\{\theta_{1}, \theta_{2}, \ldots, \theta_{n}\right\}$ um conjunto de dados, então:

(i) Estimar $\mu$ e $\kappa$ da distribuição de von Mises por meio de (3), (16) e (20);

(ii) Calcular de $z_{i}=\left.F_{\Theta}\left(\theta_{i}-\hat{\mu}\right)\right|_{\kappa=\hat{\kappa}}, \quad i=1, \ldots, n$;

(iii) Colocar $z_{i}$ em ordem crescente de modo a obter

$$
z_{[1]} \leq z_{[2]} \leq z_{[3]} \leq \ldots \leq z_{[n]}
$$

(iv) Calcular o valor da estatística $U^{2}$ dada por

$$
U^{2}=\sum_{i=1}^{n}\left[z_{[i]}-\frac{2 i-1}{2 n}\right]^{2}-n\left(\bar{z}-\frac{1}{2}\right)^{2}+\frac{1}{12 n}
$$

sendo $\bar{z}=\frac{1}{n} \sum_{i=1}^{n} z_{i}$

(v) Comparar o valor da estatística calculada no item anterior com o valor crítico apresentado por Lockhart \& Stephens (1985, Tabela 1) e reproduzida por Fisher (1995, p.230). 


\section{RESULTADOS E DISCUSSÃO}

De uma forma resumida, a análise dos dados apresentados nas Tabelas 1 e 2 foi feita em três etapas. Inicialmente, foram obtidas algumas medidas de locação e de concentração. Em seguida, verificou-se se os dados seguem distribuições de von Mises, foi feito o teste de homogeneidade dos parâmetros de concentrações de Mardia (1972) e posteriormente, o teste de igualdade dos ângulos médios pelo método de Stephens (1972) e de McGuire (1992) para dados de tonalidade da cor.

A Tabela 6 apresenta a média aritmética dos ângulos, o ângulo médio, o raio médio e a estimativa do parâmetro de concentração para os dados simulados de tonalidade de cor relativos a três tratamentos. De uma forma geral, os ângulos médios para os três tratamentos foram próximos aos valores populacionais $\mu_{1}=0^{0}$, $\mu_{2}=10^{\circ}$ e $\mu_{3}=355^{\circ}$.

Tabela 6. Média aritmética $\left(\bar{\theta}_{j}\right)$, ângulo médio $\left(\hat{\mu}_{j}\right)$, raio médio $\left(\bar{R}_{j}\right)$ e estimativa do parâmetro de concentração $\left(\hat{\kappa}_{j}\right)$ para os dados simulados de tonalidade de cor apresentados na Tabela 1 .

\begin{tabular}{crrrr}
\hline Tratamento $(j)$ & \multicolumn{1}{c}{$\bar{\theta}_{j}$} & \multicolumn{1}{c}{$\hat{\mu}_{j}$} & $\bar{R}_{j}$ & \multicolumn{1}{c}{$\hat{\kappa}_{j}$} \\
\hline 1 & $216,19^{0}$ & $0,19^{0}$ & 0,9968 & 155,47 \\
2 & $43,25^{0}$ & $7,26^{0}$ & 0,9962 & 132,46 \\
3 & $334,09^{0}$ & $352,09^{0}$ & 0,9971 & 171,08 \\
\hline Geral & $\bar{\theta}=197,84^{0}$ & $\hat{\mu}=359,84^{0}$ & $\bar{R}=0,9909$ & $\hat{\kappa}=55,01$ \\
& & & $\bar{R}^{\prime}=0,9967$ & $\tilde{\kappa}=151,31$ \\
\hline
\end{tabular}

Para o tratamento 1 , observa-se um ângulo médio igual a $0,19^{0}$, que 
corresponde a uma tonalidade de vermelho, condizente com os dados simulados, todos de tonalidade vermelha. Entretanto, a média aritmética para os dados desse mesmo tratamento foi $216,19^{\circ}$, correspondendo a uma tonalidade de cor entre o verde e o azul. Caso semelhante ocorre para o segundo tratamento, em que a média aritmética das tonalidades $43,25^{\circ}$ está entre o amarelo e o vermelho enquanto que todos os dados são de tonalidades vermelhas. Este fato serve para ilustrar o grave erro que se pode cometer quando, como sugere McGuire (1992), são utilizados métodos usuais de análise exploratória de dados sem levar em consideração que se tratam de dados circulares.

A Figura 9 serve para ressaltar o problema levantado, mostrando que para nenhum dos tratamentos a média aritmética das tonalidades esteve dentro do menor arco que contém todos os pontos. Mostra, ainda, que os dados apresentam, para os três tratamentos, concentrações semelhantes.
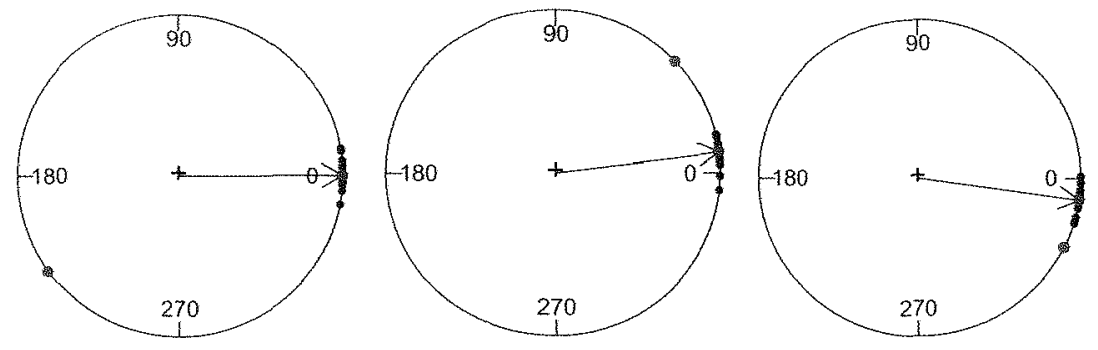

Figura 9 - Gráfico de dispersão angular para os três tratamentos simulados, respectivamente, com a indicação do vetor de comprimento igual ao raio médio, ângulo médio, em azul, média aritmética dos ângulos em vermelho

A fim de verificar, empiricamente, a hipótese de que as amostras foram extraídas de populações com distribuições de von Mises com parâmetros de concentrações iguais, construiu-se o gráfico quantil-quantil apresentado na Figura (10), a partir do qual conclui-se pela não rejeição da hipótese.

A fim de testar a hipótese de igualdade dos parâmetros de concen- 


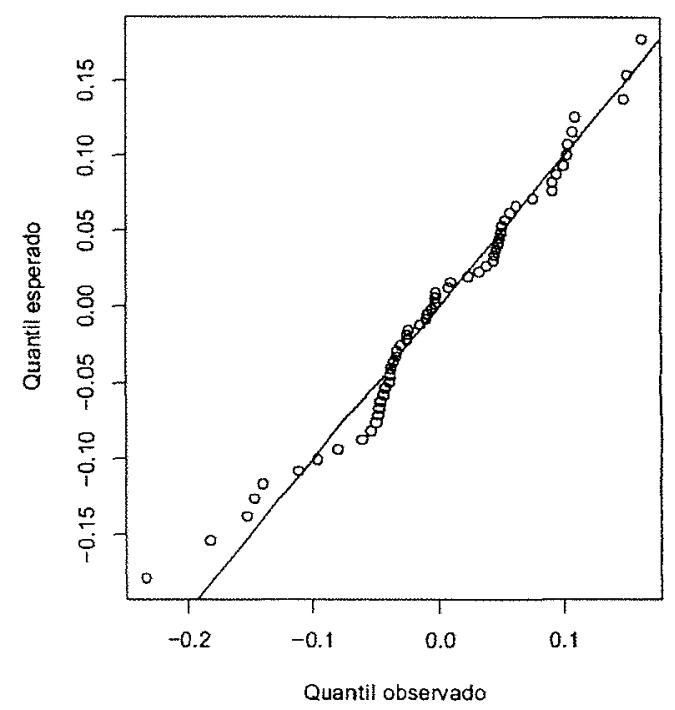

Figura 10 - Gráfico quantil-quantil para os dados de tonalidade de cor simulados, apresentados na Tabela 1

tração, $H_{0}: \kappa_{1}=\kappa_{2}=\kappa_{3}$, utilizou-se o terceiro caso da metodologia descrita. Isto devido ao fato de $\vec{R}^{\prime}=0,9967$ ser maior do que 0,70 . O valor da estatística calculada para a amostra foi $U_{3}=0,314$ e como $\operatorname{Pr}\left(\chi_{2}^{2}>0,314\right)=0,855$ é maior do que o nível de significância $\alpha=5 \%$ considerado, não se rejeita $H_{0}$. Logo, pode-se utilizar a metodologia de Stephens (1972) para testar a hipótese da igualdade dos ângulos médios $\left(H_{0}: \mu_{1}=\mu_{2}=\mu_{3}\right)$, cujos resultados são apresentados na Tabela 7. Como o valor da estatística $F=50,25$ é maior do que o valor tabelado $F_{\{2 ; 57 ; 0,05\}}=3,16$, ou o valor-p é menor do que 0,05 , rejeita-se $H_{0}$ considerando-se um nível de significância $5 \%$. Assim, há evidência estatística de que pelo menos dois tratamentos difiram entre si com relação ao ângulo médio.

Comparando-se o quadro da análise de variância apresentado na Tabela 8 com o quadro apresentado na Tabela 7 para os mesmos dados, observa-se uma grande discrepância entre os valores das estatísticas $F$, que, embora sejam obtidas 
Tabela 7. Quadro da análise de variância para os dados da Tabela 1, dados simulados, quando o método proposto for o de Stephens (1972) para dados circulares

\begin{tabular}{lccccc}
\hline CV & GL & SQ & QM & F & valor-p \\
\hline Tratamentos & 2 & 0,3493 & 0,1746 & 50,25 & 0,0000 \\
Resíduo & 57 & 0,1986 & 0,0035 & & \\
\hline Total & 59 & 0,5479 & & & \\
\hline
\end{tabular}

de formas diferentes, podem sugerir a presença problemas.

Tabela 8. Quadro da análise de variância para os dados da Tabela 1, dados simulados, em graus, quando se utiliza o método usual

\begin{tabular}{lrrrrr}
\hline CV & GL & SQ & QM & F & valor-p \\
\hline Tratamentos & 2 & 855952,7 & 427976,35 & 26,19 & 0,0000 \\
Resíduo & 57 & 931307,7 & 16338,732 & & \\
\hline Total & 59 & 1787260,4 & & & \\
\hline
\end{tabular}

O quadro da análise de variância considerando dados circulares na reta real Tabela 8 , pode esconder problemas graves no que se refere, por exemplo, às pressuposições para a análise de variância. No caso em questão, há uma severa falta de normalidade. Considerando-se, por exemplo, os dados relativos ao primeiro tratamento, pouco mais da metade estão entre $349^{\circ}$ e $360^{\circ}$ e os restantes, entre $0^{0}$ e $10^{\circ}$. Essa falta de normalidade pode ser revelada tanto por meio de histogramas (Figura 11), como por meio de testes de normalidade como o de Shapiro-Wilks cujas estatísticas para os três tratamentos foram, $W=0,638, W=0,381$ e $W=0,279$, respectivamente, todas com valores-p, menores do que 0,0001 , revelando falta de normalidade com um nível de significância de $5 \%$.

Embora possa parecer um caso específico, este fato pode ocorrer sempre que se estiver trabalhando com ângulos ao redor de $0^{0}$, que correspondem, no caso 

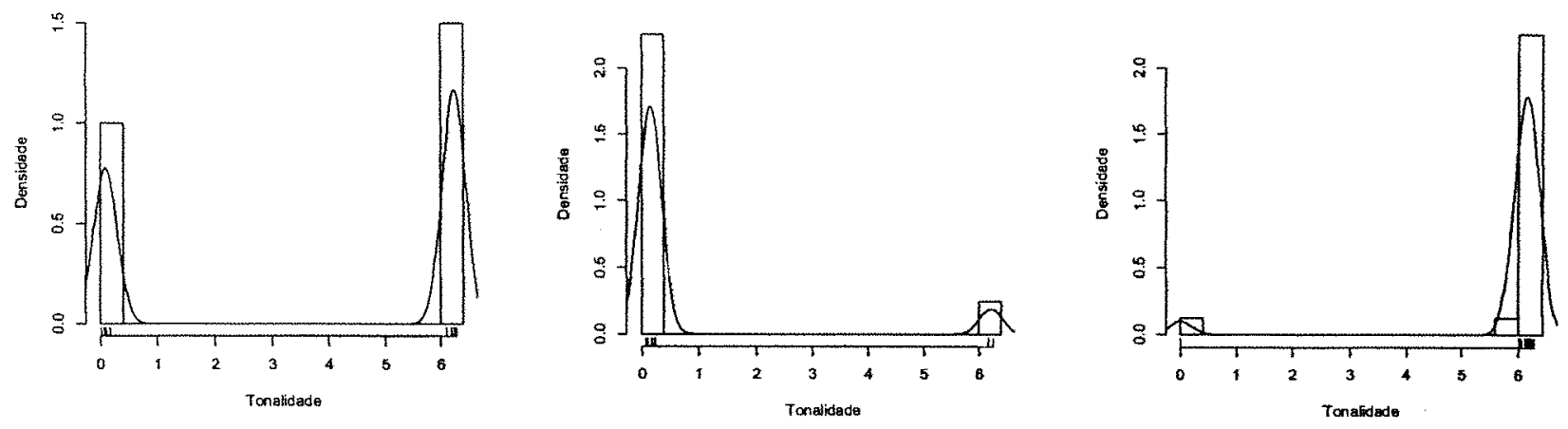

Figura 11 - Histogramas e densidades não-paramétricas para os dados de tonalidade de cor, em radianos, referentes aos três tratamentos simulados, respectivamente

de tonalidade de cores, às tonalidades avermelhadas, freqüentemente observadas em frutas e hortaliças. Suponha, agora, que se queira comparar os ângulos médios dois a dois, ou seja, determinar quais tratamentos que diferem entre si. Os resultados dessas comparações são apresentados na Tabela 9, apartir dos quais conclui-se que os ângulos médios dos tratamentos, $\mu_{1}, \mu_{2}$ e $\mu_{3}$ diferem entre si com um nível de significância $\alpha=5 \%$.

Tabela 9. Resultados do teste de comparações múltiplas aplicados aos dados da Tabela 1

\begin{tabular}{cccccc}
\hline Tratamentos & $\begin{array}{c}\hat{A} \text { Angulos } \\
\text { médios }\end{array}$ & $\begin{array}{c}\text { Diferença } \\
\text { absoluta }\end{array}$ & Estatística & valor-p pj $_{j j^{\prime}}$ \\
$\dot{j}_{i} j^{\prime}$ & $\hat{\mu}_{j}$ & $\hat{\mu}_{j^{\prime}}$ & $d_{j j^{\prime}}$ & $F_{j j^{\prime}}$ & \\
\hline 1,2 & 0,19 & 7,26 & 7,07 & 21,81 & 0,0001 \\
1,3 & 0,19 & 352,09 & 8,10 & 28,65 & 0,0000 \\
2,3 & 7,26 & 352,09 & 15,17 & 100,34 & 0,0000 \\
\hline
\end{tabular}

Considere, agora, os dados de tonalidade de cor de cogumelo no tempo zero, apresentados na Tabela 2. A Tabela 10 apresenta, para esses dados, a média 
aritmética, o ângulo médio, o raio médio e a estimativa do parâmetro de concentração. Analisando-se essas estatísticas, verifica-se que os ângulos médios são iguais às médias aritméticas, não revelando os problemas evidenciados anteriormente. Isto ocorre devido aos dados terem concentrações bastante elevadas e principalmente pelo fato de as tonalidades do cogumelo serem amarelas, isto é, revelam valores de ângulos distantes do ângulo $0^{0}$. O mesmo ocorre analisando-se os dados no tempo dez, resumidos na Tabela 11.

Tabela 10. Média aritmética $\left(\bar{\theta}_{j}\right)$, ângulo médio $\left(\hat{\mu}_{j}\right)$, raio médio $\left(\bar{R}_{j}\right)$ e estimativa do parâmetro de concentração $\left(\hat{\kappa}_{j}\right)$ para dados de tonalidade de cor de cogumelo no tempo zero, apresentados na Tabela 2

\begin{tabular}{lrrrr}
\hline Tratamento $(j)$ & $\bar{\theta}_{j}$ & $\hat{\mu}_{j}$ & $\bar{R}_{j}$ & $\hat{\kappa}_{j}$ \\
\hline 1. Testemunha & $94,76^{0}$ & $94,76^{0}$ & 0,9999 & 2941,26 \\
2. Peróxido de hidrogênio & $98,63^{0}$ & $98,63^{0}$ & 0,9998 & 1624,64 \\
3. Água destilada & $97,38^{0}$ & $97,38^{\circ}$ & 0,9999 & 1895,24 \\
4. Ácido cítrico & $101,24^{0}$ & $101,24^{0}$ & 0,9994 & 433,08 \\
\hline Geral & $\bar{\theta}=98,01^{0}$ & $\hat{\mu}=98,01^{0}$ & $\bar{R}=0,9989$ & $\hat{\kappa}=471,16$ \\
& & & $\bar{R}^{\prime}=0,9998$ & $\tilde{\kappa}=2142.39$ \\
\hline
\end{tabular}

Verifica-se, ainda, que para os dados no tempo zero, $\bar{R}^{\prime}=0,9998>$ 0,75 cabendo a utilização da terceira alternativa da metodologia para a avaliação da hipótese de homogeneidade dos parâmetros de concentração, cujo valor-p associado à estatística $U_{3}=4,18$ é 0,243 . Não se rejeita, portanto, essa hipótese, com um nível de significância $\alpha=5 \%$. Para os dados no tempo dez, tendo em vista que $\bar{R}=0,9994>0,75$, utilizou-se a mesma alternativa da metodologia, obtendo-se o valor da estatística $U_{3}=6,1760$, com valor-p igual a 0,103 , chegando-se à mesma conclusão.

Logo, pode-se utilizar a metodologia de Stephens (1972) para testar a hipótese da igualdade dos ângulos médios $\left(H_{0}: \mu_{1}=\cdots=\mu_{4}\right)$, cujos resultados 
Tabela 11. Média aritmética $\left(\bar{\theta}_{j}\right)$, ângulo médio $\left(\hat{\mu}_{j}\right)$, raio médio $\left(\bar{R}_{j}\right)$ e estimativa do parâmetro de concentração $\left(\hat{\kappa}_{j}\right)$ para dados de tonalidade de cor de cogumelo no tempo dez, apresentados na Tabela 2

\begin{tabular}{lcrrr}
\hline Tratamento $(j)$ & $\bar{\theta}_{j}$ & $\hat{\mu}_{j}$ & $\bar{R}_{j}$ & $\hat{\kappa}_{j}$ \\
\hline 1. Testemunha & $90,09^{\circ}$ & $90,09^{0}$ & 0,9999 & 7185,10 \\
2. Peróxido de hidrogênio & $90,28^{0}$ & $90,28^{0}$ & 0,9995 & 1038,02 \\
3. Água destilada & $90,76^{0}$ & $90,76^{0}$ & 0,9994 & 862,69 \\
4. Ácido cítrico & $93,79^{0}$ & $93,79^{0}$ & 0,9987 & 378,73 \\
\hline Geral & $91,23^{0}$ & $91,23^{0}$ & 0,9990 & 523,88 \\
& $\bar{\theta}=91,23^{0}$ & $\hat{\mu}=91,23^{0}$ & $\bar{R}^{\prime}=0,9994$ & $\tilde{\kappa}=815,99$ \\
\hline
\end{tabular}

são apresentados nas Tabelas 12 e 13 para os dados relativos aos tempos zero e dez, respectivamente. Assim, baseando-se nos valores-p apresentados nessas tabelas, rejeita-se $H_{0}$ para os dados relativos ao tempo zero e não se rejeita $H_{0}$ para os dados relativos ao tempo dez, com um nivel de significância $\alpha=5 \%$.

Tabela 12. Quadro da análise de variância para os dados de tonalidade de cor de cogumelo no tempo zero, apresentados na Tabela 2, usando o método proposto por Stephens (1972) para dados circulares

\begin{tabular}{lccccc}
\hline CV & GL & SQ & QM & F & valor-p \\
\hline Tratamentos & 3 & 0,0166 & 0,0055 & 18,93 & 0,0000 \\
Resíduo & 16 & 0,0047 & 0,0003 & & \\
\hline Total & 19 & 0,0213 & & & \\
\hline
\end{tabular}

De modo a determinar quais são os tratamentos que diferem entre si, calculou-se para cada par de médias de tratamentos a estatística $F$ de acordo com a equação (25). Apartir dos resultados apresentados na Tabela 14, conclui-se, com um nível de significância $\alpha=5 \%$, que os ângulos médios relativos aos tratamentos 1 e 2, 1 e 4 e 3 e 4 diferem entre si. 
Tabela 13. Quadro da análise de variância para os dados de tonalidade de cor de cogumelo no tempo dez, apresentados na Tabela 2, usando o método proposto por Stephens (1972) para dados circulares

\begin{tabular}{lccccc}
\hline CV & GL & SQ & QM & F & valor-p \\
\hline Tratamentos & 3 & 0,0068 & 0,0023 & 2,98 & 0,06283 \\
Resíduo & 16 & 0,0123 & 0,0008 & & \\
\hline Total & 19 & 0,0191 & & & \\
\hline
\end{tabular}

Tabela 14. Resultados do teste de comparações múltiplas aplicados aos dados da Tabela 2

\begin{tabular}{cccccc}
\hline Tratamentos & \multicolumn{2}{c}{$\begin{array}{c}\text { Angulos } \\
\text { médios }\end{array}$} & $\begin{array}{c}\text { Diferença } \\
\text { absoluta }\end{array}$ & Estatística & valor- $p_{j j^{\prime}}^{*}$ \\
$j, j^{\prime}$ & $\hat{\mu}_{j}$ & $\hat{\mu}_{j^{\prime}}$ & $d_{j j^{\prime}}$ & $F_{j j^{\prime}}$ & \\
\hline 1,2 & 94,76 & 98,63 & 3,87 & 19,53 & 0,0020 \\
1,3 & 94,76 & 97,38 & 2,62 & 8,96 & 0,0460 \\
1,4 & 94,76 & 101,24 & 6,48 & 54,76 & 0,0000 \\
2,3 & 98,63 & 97,38 & 1,25 & 2,04 & 0,6747 \\
2,4 & 98,63 & 101,24 & 2,61 & 8,89 & 0,0471 \\
3,4 & 97,38 & 101,24 & 3,86 & 19,43 & 0,0020 \\
\hline
\end{tabular}

Comparando-se os valores das estatísticas $F$ apresentados nas Tabelas 12 e 13, geradas utilizando-se o método proposto por Stephens (1972), com os respectivos valores apresentados nas Tabelas 15 e 16, geradas utilizando-se o método usual de análise de variância, verifica-se que são bastante próximos e levam às mesmas conclusões. Isso se deve ao fato de os comprimentos dos raios médios envolvidos $(\bar{R})$ serem muito próximos a 1, e portanto a distribuição von Mises aproxima-se da distribuição normal.

Foi visto que para a análise dos dados de cogumelo as duas metodologias foram equivalentes. Porém, mesmo quando a distribuição de von Mises se 
Tabela 15. Quadro da análise de variância para os dados de tonalidade de cor de cogumelo no tempo zero, em graus, apresentados na Tabela 2, usando o método tradicional

\begin{tabular}{lrrrrr}
\hline CV & GL & SQ & QM & F & valor-p \\
\hline Tratamentos & 3 & 108,845 & 36,282 & 18,93 & 0,0000 \\
Resíduo & 16 & 30,663 & 1,916 & & \\
\hline Total & 19 & 139,508 & & & \\
\hline
\end{tabular}

Tabela 16. Quadro da análise de variância para os dados de tonalidade de cor de cogumelo no tempo dez, em graus, apresentados na Tabela 2, usando o método tradicional

\begin{tabular}{lrrrrr}
\hline CV & GL & SQ & QM & F & valor-p \\
\hline Tratamentos & 3 & 44,928 & 14,976 & 2,97 & 0,0629 \\
Resíduo & 16 & 80,524 & 5,033 & & \\
\hline Total & 19 & 125,452 & & & \\
\hline
\end{tabular}

aproxima da distribuição normal e não se detecta falta de normalidade, um problema grave pode surgir ao se utilizarem métodos de comparações múltiplas para as médias aritméticas de dados de tonalidade de cor, como sugerido por McGuire (1992).

Suponha que por um método de comparações múltiplas qualquer, a diferença mínima significativa entre duas médias seja $15^{0}$ e que se obtenham as seguintes médias de tratamentos: $\bar{\theta}_{1}=5^{0}, \bar{\theta}_{2}=9^{0}$ e $\bar{\theta}_{3}=355^{\circ}$. Nesse caso, poderíamos concluir, erroneamente, que há dois grupos de tratamentos distintos, o primeiro formado pelos dois primeiros tratamentos e o segundo formado apenas pelo terceiro, quando, na verdade, não há diferença entre eles, quando se consideram as médias na circunferência.

Assim, uma vez que a metodologia para análise de dados circulares é bastante simples e muito fácil de ser implementada computacionalmente, e que a 
metologia sugerida por McGuire (1992) apresenta problemas graves por não levar em conta a periodicidade do círculo, sugere-se que esta última não deva ser utilizada. 


\section{CONCLUSÕES}

As análises efetuadas no presente trabalho permitem concluir que:

A metologia sugerida por McGuire (1992) para a análise de dados de tonalidade de cor de frutas e hortaliças apresenta graves problemas por não levar em conta o fato de a tonalidade ser uma medida circular, e portanto periódica. A aplicação desse método, considerando esse aspecto como se fosse uma medida pertencente à reta real, e os dados como tendo a distribuição normal, apresenta estimativas inconsistentes para medidas de tonalidade de cor, podendo levar a obtenção de resultados não compatíveis com as amostras analisadas.

A metodologia para análise variância de dados circulares provenientes de experimentos inteiramente ao acaso com um fator proposta por por Watson \& Williams (1956) e modificada posteriormente por Stephens (1972), apresenta-se como a mais apropriada para esse tipo de análise, com resultados consistentes pois leva em conta a periodicidade do círculo e seus métodos de estimação, muito simples de ser utilizada e facilmente implementada computacionalmente.

Por esses motivos conclui-se que a metologia sugerida por McGuire (1992) não é apropriada para a análise de dados de tonalidade de cor com um fator, sendo a de Stephens (1972) a mais adequada. 


\section{REFERÊNCIAS BIBLIOGRÁFICAS}

ABRAMOWITZ, M.; STEGUN, I.A., Handbook of mathematical functions. New York: Dover, 1965.

ADOBE SYSTEMS, Color and color management technical guides: color models. http://www.adobe.com/support/techguides/color/ (13 may 2003)

AGOSTINELLI, C.; The CirStats Package version 0.1-5. http://cran.r-project.org/ (07 dec. 2002).

BATSCHELET, E. Circular statistics in biology. London: Academic Press, 1981. $387 \mathrm{p}$.

BEST, D.J.; FISHER, N.I. The bias of the maximum likehood estimators of the von Mises-Fisher concentration parameters. Communications in Statistics Simulation and Computation, 10, p.493-502, 1981.

BIBLE, B.B.; SINGHA, S. Canopy position influences CIELAB coordinates of peach color. HortScience, v.28, n.10, p.992-993, 1993.

CLYDESDALE, F.M. Color as a factor in food choice. Critical Review Food Sciences and Nutrition, v.33, n.1, p.83-101, 1993.

D'SOUZA, M.C.; SINGHS, S.; INGLE, M. Lycopene concentration of tomato fruit can be estimaded from chromaticity values. HortScience, v.27, n.5, p.465-466, 1992. 
FISHER, N.I. Statistical analysis of circular data. Cambridge: Cambridge University Press, 1995. 295p.

FRANCIS, F.J. Color quality evaluation of horticultural crops. HortScience, v.15, n.1, p.58-59, 1980.

FRANCIS, F.J. Colorimetric properties of foods. In: JOWITT, R.J.; ESCHER, E.; KENT, M.; McKENNA, B. (Ed.). Physical properties of foods - 2, London: Elsevier Applied Science, 1987. p.380-422.

FRANCIS, F.J. Color analysis. In: NIELSEN, S.S. (Ed.). Food analysis, 2.ed. Maryland: Aspen Publishers, 1998. p.599-612.

FRANCIS, F.J. Color measurement and interpretation. In: FUNG, D.Y.C. (Ed.). Instrumental methods for quality assurance in foods, New York: Marcel Dekker, 1998a. p.524-544.

HARRISON, D.; KANGI, G.K.; GADSEN, R.J. Analysis of variance for circular data. Journal of Applied Statistics, v.13, n.2, p.123-138, 1986.

LOCKHART, R.A.; STEPHENS, M.A. Tests of fits for the von Mises distribuition Biometrika, v.72, p.647-652, 1985.

MARDIA, K.V. Statistics of data directional. London: Academic Press, 1972. $377 p$.

MARDIA, K.V. Directional data analysis: an overview. Journal of Applied Statistics, v.15, n.2, p.115-122, 1988.

MCGUIRE, R.G. Reporting of objective color measurements. HortScience, v.27, n.12, p.1254-1255, 1992. 
MODA, E.M. Produção de pleurotus sajor-caju em bagaço de cana-de açúcar lavado e o uso de aditivos visando sua conservacão in natura. Piracicaba, 2003. 84p. Dissertação (Mestrado) - Escola Superior de Agricultura "Luiz de Queiroz", Universidade de São Paulo.

STEPHENS, M.A. Exact and approximate tests for direction I. Biometrika, v.49, p.463-477, 1962a.

STEPHENS, M.A. Exact and approximate tests for direction II. Biometrika, v.49, p.547-552, $1962 b$.

STEPHENS, M.A. Multisample tests for the von Mises distribution. Journal of the American Statistical Association, v.67, n.338, p.456-461, 1972.

STEPHENS, M.A. Use of the von Mises distribuition to analyse continuous proportions. Biometrika, v.69, p.197-203, 1982.

UPTON, G.J.G. New approximations to the distribution of certain angular statistics. Biometrika, v.61, p.369-373, 1974.

VENABLES, W.N.; SIMITH, D.M. An introduction to R Notes on R: A Programming environment for data analysis and graphics version 1. http://cran.rproject.org/ (17 june 2002.)

WATSON, G.S.; WILLIAMS, E.J. On the construction of significance tests on the circle and the sphere. Biometrika, v.33, p.344-352, 1956.

ZAR, J.H. Biostatistical analysis. 4.ed. New Jersey: Prentice Hall, 1999. 929p. 